\title{
Factor models with many assets: strong factors, weak factors, and the two-pass procedure
}

\author{
by Stanislav Anatolyev1 and Anna Mikusheva2
}

\begin{abstract}
This paper re-examines the problem of estimating risk premia in unconditional linear factor pricing models. Typically, the data used in the empirical literature are characterized by weakness of some pricing factors, strong cross-sectional dependence in the errors, and (moderately) high cross-sectional dimensionality. Using an asymptotic framework where the number of assets/portfolios grows with the time span of the data while the risk exposures of weak factors are local-to-zero, we show that the conventional two-pass estimation procedure delivers inconsistent estimates of the risk premia. We propose a new estimation procedure based on sample-splitting instrumental variables regression. The proposed estimator of risk premia is robust to weak included factors and to the presence of strong unaccounted cross-sectional error dependence. We derive the many-asset weak factor asymptotic distribution of the proposed estimator, show how to construct its standard errors, verify its performance in simulations, and revisit some empirical studies.

Key words: factor models, price of risk, risk premia, two-pass procedure, strong factors, weak factors, dimensionality asymptotics, weak factor asymptotics.

JEL classification codes: C33, C38, C58, G12.
\end{abstract}

\section{Introduction}

Since the introduction of the CAPM by Sharpe (1964) and Linner (1965), linear factor pricing models have grown into a very popular sub-field in asset pricing. Harvey, Liu and Zhu (2016) list hundreds of papers that propose, justify and estimate various factor pricing models. A typical paper in this area proposes a small set of observed risk factors that price

\footnotetext{
${ }^{1}$ CERGE-EI and New Economic School. Address: Politických vězňů 7, 11121 Prague, Czech Republic. E-mail: stanislav.anatolyev@cerge-ei.cz. Czech Science Foundation support under grant number 17$26535 \mathrm{~S}$ is gratefully acknowledged.

2 Department of Economics, M.I.T. Address: 77 Massachusetts Avenue, E52-526, Cambridge, MA, 02139. Email: amikushe@mit.edu. National Science Foundation support under grant number 1757199 is gratefully acknowledged. We thank David Hughes for research assistance.
} 
the assets, that is, the expected excess return on an asset is equal to the quantity of risk taken (measured as a normalized covariance of the returns with the risk factors, so called betas) times risk premia. The two most famous factor pricing models are the market-factor CAPM and the three-factor Fama and French (1993) model. Other pricing factors are the momentum factor (Jegadeesh and Titman, 1993), the consumption-to-wealth ratio 'cay' (Lettau and Ludvigson, 2001), the liquidity factor (Pástor and Stambaugh, 2003), and so on. In recent years, there has been a burst in econometrics research that suggests how to correct the baseline estimation and inference in the face of many factors (for example, Kozak, Nagel, and Santosh, 2018), or how to judiciously select factors from a big pool without jeopardizing correct inference (for example, Feng, Giglio and Xiu, 2019).

Traditionally, one estimates the model using what is commonly known as the two-pass estimation procedure (Fama and MacBeth, 1973; Shanken, 1992) 3 where at the first pass one estimates risk exposures (betas) for each asset, and then, at the second pass, those estimates are used as regressors to estimate the risk premia. Asymptotic justification of this procedure, however, relies on assumptions that often do not hold up in realistic circumstances. Two types of violations of the idealistic setting have been noted in previous literature.

The first problem is one of weak (but priced) observed factors. Recent papers by Kan and Zhang (1999), Kleibergen (2009), Bryzgalova (2016), Burnside (2016), and Gospodinov, Kan and Robotti (2017) all point out that risk exposures (or betas) to some observed factors tend to be small to such an extent that their estimation errors are of the same order of magnitude as the betas themselves. This observed phenomenon is very similar to the widely studied weak instrument problem.

The second violation is a strong cross-sectional dependence in error terms, which in many cases can be modeled as a factor structure unaccounted for ('missing'). For example, recent literature shows that mismeasurement of the true risk factors leads to weakness of the observed factors and strong cross-sectional dependence in the errors (Kleibergen

\footnotetext{
${ }^{3}$ Sometimes the two-pass procedure is referred to as the Fama-MacBeth procedure (Fama and MacBeth, 1973). See Cochrane (2001, section 12.3) on their numerical equivalence when betas are time invariant. The method for obtaining valid standard errors that account for the two step nature of the procedure is given in Shanken (1992).
} 
and Zhan, 2015), which may result in all sorts of distortions in estimation and inference in theory and in their non-reliability in practice (Kan and Zhang, 1999; Andrews, 2005; Kleibergen, 2009).

Along with the combination of the problems of missing factors and small betas, we also consider one very important empirical feature of the typically employed datasets the presence of a large number of assets or portfolios often comparable to the number of periods over which returns are observed. We consider an asymptotic framework where the number of assets/portfolios grows with its time-series dimension. Such dimension asymptotics is likely to provide a more accurate asymptotic approximation to the finite sample properties of estimators and tests. The many-asset asymptotic framework has been utilized previously by Gagliardini, Ossola and Scaillet (2016a), Lettau and Pelger (2018) and Feng, Giglio and Xiu (2019).

We show that within a dimension-asymptotic framework the presence of small betas leads to a failure of the classical two-pass procedure, while the additional presence of missing factors exacerbates this problem. We propose econometric procedures that are robust to both these thorny issues with factors - the weakness of observed factors and the presence of unobserved factors in the errors - and, in contrast to the remedies proposed elsewhere, are easily implementable using standard regression tools (in particular, instrumental variables regressions and two-stage least squares). The estimators we propose are consistent and asymptotically mixed gaussian; moreover, using the variance estimators (the construction of which we describe), standard inference tools such as $t$ - and Wald tests can be applied in a conventional way.

Our new estimation approach makes use of the idea of sample-splitting in order to create multiple estimates for loadings $\beta_{i}$ and to correct for the first-step estimation error via an instrumental variables regression. The presence of an unobserved (missing) factor structure in the error terms creates strong cross-sectional dependence in the panel of returns, which is similar to the classical omitted-variables problem in the second pass of the two-pass procedure. In order to correct for this missing factor structure, we use sample splitting to create reasonable proxies for missing factors even in a setting where one cannot consistently estimate the missing factor structure. The sample-splitting idea 
has appeared in the econometrics literature before, in particular, in Angrist and Krueger (1992) and Dufour and Jasiak (2001).

We explore the quality of the two-pass procedure and compare its performance with that of sample-splitting based estimators in simulations calibrated to match the monthly returns of the 100 Fama-French sorted portfolios. We applied both procedures to the estimation of the momentum risk premium using real data on Fama-French portfolios. The important feature here is that the momentum is a tradable factor and hence there is an alternative estimate of the risk premia - the sample average excess return on this factor. Thus, we have a natural benchmark when comparing two estimators, which differ greatly. Lewellen, Nagel and Shanken (2010) showed that "any (sufficiently large)4 set

of assets perfectly explains the cross-section of expected returns so long as the (tested) 5 assets are not asked to price themselves (that is, ...the risk premia are not required to equal their expected returns)." From that perspective, having an estimate of risk premia coming from the pricing model, such as our split-sample estimator, is important even for tradable factors, as it allows one to test the pricing model by comparing this estimate to the average excess return.

There is a growing number of alternative suggestions for how to correct statistical inferences for either weak observed factors or a missing factor structure, but we are not aware of any paper that solves both problems simultaneously. Kleibergen (2009) proposes the use of weak identification robust inference procedures to account for weak observed factors, however, it can only be applied to a relatively small number of assets/portfolios. An extreme version of the weak factors phenomenon, known as irrelevant factors and studied by Bryzgalova (2016), Burnside (2016) and Gospodinov, Kan and Robotti (2017), occurs when some observed factors are assumed to have zero loadings. The solutions to the irrelevant factors problem proposed in the literature usually suggest dimension reduction techniques to detect the irrelevant factors with a proviso to eliminate these detected irrelevant factors from further analysis. However, applying detection and elimination methods to the weak observed factors would lead to invalid inferences and large

\footnotetext{
${ }^{4}$ Our addition in brackets.

${ }^{5}$ Our addition in brackets.
} 
biases in the estimates of the risk premia for the remaining factors. Jegadeesh, Noh, Pukthuanthong, Roll and Wang (2019) suggest, simultaneously and independently, the use of sample-splitting in factor models in order to fix the errors-in-variables bias. Their proposed estimator works only when there are no missing factors in the error terms.

Giglio and Xiu (2017) solve the problem of the missing factor structure by first running the Principle Component Analysis (PCA) on excess returns, pricing principle components, and from that deriving the risk premia of observed factors. The method of Giglio and Xiu (2017) successfully eliminates strong missing factors, but assumes from the outset that all important pricing factors can be uncovered by PCA - essentially, they assume away any existence of weak factors needed for pricing. This assumption is critical for the validity of their procedure and contradicts the empirical findings of Lettau and Pelger (2018), who demonstrate that the out-of-sample performance using weak factors in addition to those uncovered by PCA is sizably better than that of a model that uses the PCA factors only. According to Lettau and Pelger (2018), "PCA-based factors often miss low volatility components with high Sharpe ratios, which is a crucial aspect in asset pricing."

The paper is organized as follows. Section 2 introduces notation, discusses the relevance of our asymptotic approach, and argues for the presence of a significant factor structure in the errors. Section 3 introduces and discusses technical assumptions. Section 4 explains the asymptotic failure of the classical two-pass procedure and provides detailed intuition as to why this occurs. We propose our 'four-split' estimation method in Section 5. describe what motivates it and explain why it works. We also state a formal theorem on the consistency of the newly-proposed four-split estimator. Section 6 is devoted to deriving inference procedures that use our four-split estimator; in particular, we show the asymptotic validity of a properly constructed Wald test. The size of a potential failure of the two-pass procedure and the behavior of the newly proposed estimator are studied in simulations and with an empirical example in Section 7 . Some proofs and additional results appear in a Supplementary Appendix available on the authors' web-sites 6

A word on notation: $0_{l, m}$ stands for a zero matrix of size $l \times m, I_{m}$ is an $m \times m$ identity matrix; for an $m \times l_{A}$ matrix $A$ and an $m \times l_{B}$ matrix $B,(A, B)$ stands for the

\footnotetext{
${ }^{6}$ http://economics.mit.edu/files/15225
} 
$m \times\left(l_{A}+l_{B}\right)$ matrix one obtains by placing the initial matrices side-to-side. For a square matrix $A$ we denote $\operatorname{dg}(A)$ a diagonal matrix of the same size with the same elements on the diagonal as matrix $A$.

\section{Formulation of the problem}

The research in factor asset pricing modeling typically proposes a small set of observed risk factors described by a vector $F_{t}$ of (usually) small dimension $k_{F}$. An asset or portfolio of assets $i$ with excess return $r_{i t}$ has exposure to several risk factors, which is quantified by the asset's betas $\beta_{i}=\operatorname{var}\left(F_{t}\right)^{-1} \operatorname{cov}\left(F_{t}, r_{i t}\right)$. A typical claim put forth in the linear factor-pricing theory is that exposure to risk (betas) fully determines the assets' expected excess returns. Particularly, there exists a $k_{F}$-dimensional vector of risk premia $\lambda$ such that $E r_{i t}=\lambda^{\prime} \beta_{i}$. This is known as unconditional factor pricing setting, and some generalizations to conditional factor pricing will be discussed later on.

From an econometric perspective, a correctly-specified linear factor-pricing model is equivalent to the following formulation:

$$
r_{i t}=\lambda^{\prime} \beta_{i}+\left(F_{t}-E F_{t}\right)^{\prime} \beta_{i}+\varepsilon_{i t},
$$

where unobserved random error terms $\varepsilon_{i t}$ have mean zero and are uncorrelated with $F_{t}$. Here the statement $E \varepsilon_{i t}=0$ is equivalent to $E r_{i t}=\lambda^{\prime} \beta_{i}$, while uncorrelatedness between $\varepsilon_{i t}$ and $F_{t}$ results from the definition of $\beta_{i}$. We treat $\lambda$ and $\beta_{i}$ as unknown parameters, while $r_{i t}, F_{t}$, and $\varepsilon_{i t}$ are random variables. One special case often mentioned in the literature occurs when the $F_{t}$ factors are asset returns themselves and are supposed to be priced by the same model; in this case, theoretically $\lambda=E F_{t}$. We will not make this assumption and will consider the general case when $\lambda$ differs from $E F_{t}$.

Two-pass procedure. The estimation and inferences on risk prices, $\lambda$, are usually accomplished by a procedure commonly known as the two-pass procedure (Fama and MacBeth, 1973; Shanken, 1992), which is applied to a data set consisting of a panel of asset excess returns $\left\{r_{i t}, i=1, \ldots, N, t=1, \ldots, T\right\}$ and observations of realized factors $\left\{F_{t}, t=1, \ldots, T\right\}$. In the first step, one estimates $\beta_{i}$ by running a time series OLS 
regression of $r_{i t}$ on a constant and $F_{t}$ for each $i=1, \ldots, N$. The second step produces an estimate of $\lambda$ (denote it $\widehat{\lambda}_{T P}$ ) by regressing the time-average excess return $\frac{1}{T} \sum_{t=1}^{T} r_{i t}$ on the first-step estimates, $\widehat{\beta}_{i}$. Under suitable conditions, $\widehat{\lambda}_{T P}$ is proved to be both consistent and asymptotically gaussian. Discussions of the statistical properties of the two-pass procedure appear in Fama and MacBeth (1973), Shanken (1992), and Chapter 12 of Cochrane (2001).

This paper deviates from the classical Fama-MacBeth setting in three respects, which we label as (i) weak observed factors, (ii) many assets and (iii) missing factor structure.

Weak observed factors. Recent work by several prominent researchers raises the concern that the two-pass procedure may provide misleading estimates of risk premia; see, for example, Kan and Zhang (1999), Kleibergen (2009), Bryzgalova (2016), Burnside (2016), Gospodinov, Kan and Robotti (2017). They surmise that the reason for these erroneous inferences is attached to the empirical observation that either some column of $\beta=\left(\beta_{1}, \ldots, \beta_{N}\right)^{\prime}$ is close to zero, or, more generally, the $N \times k_{F}$ matrix $\beta$ appears close to one of reduced rank (less than $k_{F}$ ) for many well-known linear factor pricing models. According to Lettau and Pelger (2018), weak factors are empirically crucial for good performance of pricing models. They constructed factors that are impossible to uncover by PCA (i.e. weak factors) with the Sharpe ratio twice as high as those uncovered by PCA, and the out-of-sample pricing errors from a model that uses these weak factors are sizably smaller than those a the model that uses only strong factors.

Bryzgalova (2016), Burnside (2016), and Gospodinov, Kan and Robotti (2017) all recently developed improved inference procedures when some factors are completely irrelevant for pricing, that is, when true $\beta_{i}$ are exactly zeros. The above-mentioned papers propose strategies for detecting and eliminating irrelevant factors, which is important as the risk premia on the irrelevant factor are completely unidentified. Unfortunately, these procedures fail when the $\beta_{i}$ are not zeros, but are rather small.

A more empirically relevant case, which is in line with Lettau and Pelger (2018), resembles the widely studied weak instrument problem (Staiger and Stock, 1998): if some of the observed factors $F_{t}$ are only weakly correlated with all the returns in the data set, then the noise that arises in the first-pass estimates of the corresponding components 
of $\beta_{i}$ will dominate the signal, and the second-pass estimate of the risk premia $\lambda$ will be oversensitive to small perturbations in the sample. In order to model the observed phenomenon, Kleibergen (2009) considered a drifting-parameter framework in which some component of $\beta_{i}$ is modeled to be of order $O\left(\frac{1}{\sqrt{T}}\right)$ assuming that the number of time periods, $T$, increases to infinity, while the number of assets, $N$, stays fixed. In such a setting the first-pass estimation error is of order of magnitude $O_{p}\left(\frac{1}{\sqrt{T}}\right)$, which is comparable to the size of the coefficients themselves. This framework implies inconsistency of the two-pass estimates for the risk premium on small components, poor coverage of regular confidence sets even for the risk premium of strong factors, and asymptotic invalidity of classical specification tests and tests about risk premia.

Following this tradition and acknowledging the empirical evidence provided in Kleibergen and Zhan (2015) and Bryzgalova (2016), we also make use of drifting-parameter modeling. We assume that the $k_{F} \times 1$ vector of factors $F_{t}$ can be divided into two subvectors: a $k_{1} \times 1$ dimensional vector $F_{t, 1}$ and a $k_{2} \times 1$ vector $F_{2, t}$ (here $\left.k_{F}=k_{1}+k_{2}\right)$ such that the risk exposure $\beta_{i, 1}$ to factor $F_{t, 1}$ will be strong, while the risk exposure coefficients $\beta_{2, i}$ to factor $F_{2, t}$ will be drifting to zero at the rate $\sqrt{T}$. We make these order assumptions for risk exposures more accurate in the next section. The most important feature of our modeling is that the standard error of the first-pass estimator of $\beta_{2, i}$ will be of the same order of magnitude as the coefficient itself. A more general treatment of the near-degenerate rank condition considers some $k_{2}$-dimensional linear combination of factors (unknown to the researcher) to have a local-to-zero (of order $O\left(\frac{1}{\sqrt{T}}\right)$ ) exposure coefficient, while the exposure to risk formed by the orthogonal $k_{1}$-dimensional linear combination remains fixed. All our results are easily generalizable to this setting, as we do not assume that the researcher knows which factors (or combination of factors) bear small coefficients of exposure. However, to simplify the exposition we will stick to the division of factors into two sub-vectors.

Many assets. In theoretical justifications of the two-step procedure (Shanken, 1992; Cochrane, 2001) it is common to assume that the number of assets, $N$, is fixed, while the number of periods $T$ grows to infinity. We notice that in many common data sets that researchers use, the number of assets is large when compared to the number of time peri- 
ods. The celebrated Fama-French data set provides returns on $N=25$ sorted portfolios for about $T=200$ periods. The often-used Jagannathan-Wang data set (Jagannathan and Wang, 1996) contains observations on $N=100$ portfolios observed for $T=330$ periods. Lettau and Ludvigson (2001) use Fama-French $N=25$ portfolios, the returns for which are observed over $T=141$ quarters. Gagliardini, Ossola and Scaillet (2016a) use $N=44$ industry portfolios observed during $T=546$ months. In these cases it is hard to believe that the asymptotic results derived under the assumption that $N$ is fixed would provide an accurate approximation of finite-sample distributions. Indeed, among other things, Kleibergen (2009) discovers that the bias of the two-pass estimate of risk premia is strongly and positively related to the number of assets if the total factor strength is kept constant.

In this paper we consider asymptotics when both $N$ and $T$ increase to infinity without restricting the relative growth between them. Two recent papers by Raponi, Robotti and Zaffaroni (2017) and Kim and Skoulakis (2018) consider a factor pricing model in an asymptotic setting with $N \rightarrow \infty$ while $T$ remains fixed. They show an inconsistency of the two-pass procedures, which arises from the fact that with fixed $T$ loadings $\beta_{i}$ are estimated from a finite sample; as a result, the estimation error of $\beta_{i}$ is non-negligible. This is exactly the same phenomenon we are modeling with weak factors when we consider $T \rightarrow \infty$ - namely, non-negligibility of the first step estimation errors. We can show that the procedures we propose are consistent in the setting with $N \rightarrow \infty$, fixed $T$ for ex-post risk premia (see Shanken, 1992, for distinction with ex-ante risk premia) if we impose slightly stronger assumptions on the cross-sectional dependence of the error terms than the one introduced in Assumptions ERRORS below.

Missing factor structure. This paper's main deviation from the existing literature is our explicit acknowledgment of high cross-sectional dependence among error terms $\varepsilon_{i t}$ in model (1). In particular, we assume that errors have a factor structure. Namely, this means that there exists, unknown and unobserved to the researcher, a factor $v_{t}$ and loadings $\mu_{i}$ such that

$$
\varepsilon_{i t}=v_{t}^{\prime} \mu_{i}+e_{i t}
$$


where the 'clean' errors $e_{i t}$ are only weakly cross-sectionally dependent to the extent that asymptotically we may ignore their dependence (the exact formulation of this assumption appears in the next section). Similar weak-dependence assumptions appear in approximate factor models (e.g., Bai and Ng, 2002). The assumptions on loadings $\mu_{i}$ guarantee that the factor structure will be strong enough to be both detected empirically and asymptotically important for inferences. An insightful discussion of weak vs strong factor structure and cross-sectional dependence appears in Onatski (2012).

Below we provide two theoretical reasons as to why we expect to encounter factor structure in many linear factor-pricing models. Then we point to empirical evidence that a missing factor structure is indeed present in some well-known factor-pricing models.

Example 1. This example is similar to the one discussed in Lewellen, Nagel and Shanken (2010). If one does not observe the true risk factors that price assets but only proxies for them, this would lead to a factor structure in errors (see Kleibergen and Zhan, 2015). Assume for a moment that the market is priced by risk premia on risk factors $G_{t}$. For expositional simplicity we assume that

$$
r_{i t}=G_{t} \beta_{i}^{G}+\varepsilon_{i t}^{G}
$$

where the shocks $\varepsilon_{i t}^{G}$ are drawn with mean zero and finite variance independently crosssectionally, and independent from $G_{t}$. Assume that $G_{t}$ is stationary with variance $\Sigma_{G}$.

Assume that the econometrician does not observe $G_{t}$, but rather has a proxy for it, $F_{t}=\alpha+\delta G_{t}+\epsilon_{t}$, where $\epsilon_{t}$ has mean zero and is uncorrelated with $G_{t}$ and shocks $\varepsilon_{i t}^{G}$. For example, $\epsilon_{t}$ may stand for a measurement error or contamination by other macro variables unconnected to asset prices. Denote the variance matrix of $\epsilon_{t}$ by $\Sigma_{\epsilon}$. If $\delta$ is a full-rank square matrix, then one can show that proxies $F_{t}$ can price assets as well as the true risk factors $G_{t}$. Indeed,

$$
\beta_{i}=\operatorname{var}\left(F_{t}\right)^{-1} \operatorname{cov}\left(F_{t}, r_{i t}\right)=\left(\delta \Sigma_{G} \delta^{\prime}+\Sigma_{\epsilon}\right)^{-1} \delta \Sigma_{G} \beta_{i}^{G}=A \beta_{i}^{G},
$$

where $A=\left(\delta \Sigma_{G} \delta^{\prime}+\Sigma_{\epsilon}\right)^{-1} \delta \Sigma_{G}$ is a full-rank square matrix. Thus,

$$
E r_{i t}=E G_{t} \beta_{i}^{G}=\lambda \beta_{i}
$$

where $\lambda=A^{-1} E G_{t}$. So we see that if the econometrician is estimating a linear factorpricing model using factors $F_{t}$, she has a correctly-specified model; however, this model 
(unlike the model with the observed factors $G_{t}$ ) has a factor structure in its error terms. Indeed, using some simple algebra one can show that equation (1) holds with

$$
\varepsilon_{i t}=\left(\Sigma_{\epsilon} \delta^{-1} \Sigma_{G}^{-1}\left(G_{t}-E G_{t}\right)-\epsilon_{t}\right)^{\prime} \beta_{i}+\varepsilon_{i t}^{G}=v_{t}^{\prime} \mu_{i}+\varepsilon_{i t}^{G} .
$$

What is interesting here is that while the factors $v_{t}$ (and the errors $\varepsilon_{i t}$ themselves) are uncorrelated with the observed factors $F_{t}$, the loadings on the error factors, $\mu_{i}$ 's, and the original loadings, $\beta_{i}$ 's, are closely related (in this particular case $\mu_{i}=\beta_{i}$ ). We will make use of this observation in our discussion of the validity of the two-pass procedure.

Example 2. Consider a situation when one of the risk factors driving asset returns is fully arbitraged and thus carries a zero risk premium. If an econometrician does not observe this factor but does have observations on all other relevant risk factors, then her linear factor-pricing model that omits the arbitraged factor may still be correctly specified, while the arbitraged factor is moved to the error term, resulting in a missingfactor structure in the errors.

Kleibergen and Zhan (2015) provide numerous pieces of empirical evidence that residuals from many well-known estimated linear factor-pricing models have non-trivial factor structures. For example, they point out that the first three principle components of the residuals from different pricing-model specifications used in the seminal paper by Lettau and Ludvigson (2001) explain from $82 \%$ to $95 \%$ of all residual variation. They also show that the largest eigenvalue of the covariance matrix of residuals in all these examples is very large and strongly separated from other eigenvalues that are bunched together. Combining these results with the theoretical results on the limiting distribution of eigenvalues from Onatski (2012), one would suspect there is at least one strong factor present in the residuals. At least five other prominent factor-pricing studies cited in Kleibergen and Zhan (2015) demonstrate similar evidence of strong factor structures left in the residuals.

Relation between factor structure and correct specification. One may wonder whether the fact that the errors $\varepsilon_{i t}$ in model (11) have a factor structure implies that the pricing model is misspecified. The answer is "no"; the linear factor pricing model describes the expectations of excess returns, while the factor structure in the errors is related to their covariances or co-movements. It is easy to see that if the risk exposure and risk premia 
on the variables $F_{t}$ price the assets, then the variables $F_{t}$ co-move the assets' returns and produce factor-structure dependence in the returns. However, not all co-movements of returns must carry non-zero risk premia; those co-movements can be placed in the error term without causing misspecification of the pricing model.

The correct specification of a pricing model requires keeping those pricing factors $F_{t, 2}$ that carry small coefficients of exposure $\beta_{2, t}$ and produce only a weak factor structure in returns in the model. Dropping such observed factors from the specification leads to asymptotically misleading inferences for both the two-pass procedure and our proposed procedure.

Remark. The literature on factor pricing distinguishes cases of tradable and nontradable factors. If a specific factor $F_{t}$ is a tradable portfolio and is supposed to be priced by the same pricing model, then $\lambda=E F_{t}$, and one can get an estimate of risk premia as the sample average of the excess returns. However, even in this case there is a value from having an alternative estimator based on the pricing equation (1). Lewellen, Nagel and Shanken (2010) showed that it is relatively easy to price the market with high crosssectional $R^{2}$ by any set of portfolios as long as their number is larger than the number of the main principle components, but only if one does not enforce that the risk premia be equal the average returns. The cross-sectional $R^{2}$ of the pricing model is much smaller if one enforces such a restriction. Thus, having an estimator of the risk premia coming from the two-step pricing logic and comparing it to the average excess return for tradable factors is a valuable test of the pricing model.

\section{$3 \quad$ Setup and assumptions}

\subsection{Model}

We consider the problem of estimation and inference on the risk premia $\lambda$ based on observations of returns $\left\{r_{i t}, i=1, \ldots, N, t=1, \ldots, T\right\}$ and factors $\left\{F_{t}, t=1, \ldots, T\right\}$ obeying a correctly-specified factor-pricing model:

$$
r_{i t}=\lambda^{\prime} \beta_{i}+\left(F_{t}-E F_{t}\right)^{\prime} \beta_{i}+v_{t}^{\prime} \mu_{i}+e_{i t}
$$


where the random unobserved factor $v_{t}$ has zero mean and is uncorrelated with $F_{t}$. The idiosyncratic error terms $e_{i t}$ also have zero mean and are uncorrelated with $F_{t}$ and $v_{t}$. Denote by $\mathcal{F}$ the sigma-algebra generated by the random variables $\left(F_{1}, \ldots, F_{T}\right)$ and $\left(v_{1}, \ldots, v_{T}\right)$. Let $\gamma_{i}^{\prime}=\left(\beta_{1 i}^{\prime}, \sqrt{T} \beta_{2 i}^{\prime}, \mu_{i}^{\prime}\right)$ and $\Gamma_{N}^{\prime}=\left(\gamma_{1}, \ldots, \gamma_{N}\right)$ be the $k \times N$ matrix, where $k=k_{F}+k_{v}$. Technically, $\gamma_{i, N, T}$ is more accurate indexing, as parameters $\gamma_{i}$ may change with the sample size as do all other features of the data generating process, but we will drop $N, T$ to reduce clutter.

Assumption FACTORS. The $k_{F} \times 1$ vector of observed factors $F_{t}$ is stationary with finite fourth moments and a full-rank covariance matrix $\Sigma_{F}$. The $k_{v} \times 1$ vector of unobserved factors $v_{t}$ is such that the following asymptotic statements hold jointly:

$$
\left(\begin{array}{c}
\frac{1}{\sqrt{T}} \sum_{t=1}^{T}\left(F_{t}-E F_{t}\right) \\
\eta_{T}=\frac{1}{\sqrt{T}} \sum_{t=1}^{T} \Sigma_{F}^{-1} \widetilde{F}_{t} v_{t}^{\prime} \\
\eta_{v, T}=\frac{1}{\sqrt{T}} \sum_{t=1}^{T} v_{t}
\end{array}\right) \Rightarrow\left(\begin{array}{c}
N\left(0, \Omega_{F}\right) \\
\eta \\
\eta_{v}
\end{array}\right)
$$

where $\operatorname{vec}(\eta) \sim N\left(0_{k_{F} k_{v}, 1}, \Omega_{v F}\right), \eta_{v} \sim N\left(0_{k_{v}, 1}, I_{k_{v}}\right)$ and $\widetilde{F}_{t}=F_{t}-\frac{1}{T} \sum_{s=1}^{T} F_{s}$.

Assumption LOADINGS. As both $N$ and $T$ increase to infinity, we have $N^{-1} \Gamma_{N}^{\prime} \Gamma_{N} \rightarrow$ $\Gamma$, where $\Gamma$ is a positive definite $k \times k$ matrix. Also assume that $\max _{N, T} \frac{1}{N} \sum_{i=1}^{N}\left\|\gamma_{i}\right\|^{4}<\infty$.

\section{Assumptions ERRORS.}

(i) Conditional on $\mathcal{F}$, the random vectors $e_{t}=\left(e_{1 t}, \ldots, e_{N t}\right)^{\prime}$ are serially independent, and $E\left(e_{t} \mid \mathcal{F}\right)=0$ for all $t$.

(ii) Let $\rho(t, s)=\frac{1}{\sqrt{N}} \sum_{i=1}^{N} e_{i t} e_{i s}$. Assume $\sup _{t} \sup _{s \neq t} E\left[\left(1+\left\|F_{t}\right\|^{4}\right)\left(\rho(s, t)^{2}+1\right)\right]<C$.

(iii) Let $S_{t}=\frac{1}{N} \sum_{i=1}^{N} e_{i t}^{2}$. Assume $\frac{\sqrt{N}}{T} \sum_{t=1}^{T} \widetilde{F}_{t} S_{t}=o_{p}(1)$ and $\frac{1}{T} \sum_{t=1}^{T} \widetilde{F}_{t} \widetilde{F}_{t}^{\prime} S_{t} \rightarrow^{p} \Sigma_{S F^{2}}$.

(iv) Let $W_{t}=\frac{1}{\sqrt{N}} \sum_{i=1}^{N} \gamma_{i} e_{i t}$. Assume $E\left[\left(1+\left\|F_{t}\right\|^{2}\right)\left\|W_{t}\right\|^{2}\right]<\infty$.

\subsection{Discussion of Assumptions}

This paper adopts an asymptotic setting when both $N$ and $T$ increase to infinity without any restriction on relative size. Obviously, in an application we have fixed values of $N$ 
and $T$, and the asymptotic framework only provides an approximation to the distribution of different statistics. A natural question is when the approximations are good enough and what specific features of the application the different asymptotic rates are trying to replicate. We employ Law of Large Numbers and Central Limit Theorems for averages where summation happens over both time series and cross-sectional indexes. There are trade-offs between how large $N$ and $T$ are needed for a good approximation and the dependence structure and/or moment restrictions. Assumption ERRORS are quite agnostic about cross-sectional dependence structure but if one imposes stricter restrictions on idiosyncratic errors, then one can get good approximations even when only one index is large. Under stricter dependence assumptions, one may prove analogues of all our statements (for example, consistency of our proposed estimator) assuming that $N \rightarrow \infty$ while $T$ is fixed, if the object of interest is ex-post risk premia, $\tilde{\lambda}$, as defined in Shanken (1992).

Assumption FACTORS. Since $v_{t}$ is a structural part of the error term we already assume it is uncorrelated with $\widetilde{F}_{t}$. One can come up with a variety of assumptions on decaying dependence and moment conditions that would guarantee some version of the Central Limit Theorem as stated in Assumption FACTORS. The restriction that the asymptotic covariance matrix of $\eta_{v}$ be the identity matrix is without a loss of generality and is just a normalization, as neither $v_{t}$ nor loadings $\mu_{i}$ are observed.

Assumption LOADINGS. In this paper we treat the loadings $\beta_{i}$ and $\mu_{i}$ as unknown non-random vectors, the true values of which may change with the sample sizes $N$ and $T$. Assumption LOADINGS characterizes the size of the loadings as the sample size increases. Notice that the loadings on the factors $F_{t, 1}$ and $v_{t}$ are treated differently than the loadings on $F_{t, 2}$. Following Onatski (2012), we will refer to the former as "strong factors" and the latter as "weak factors." The cross-sectional average of squared loadings is closely connected to the explanatory power the factors exhibit in cross-sectional variation. The assumptions we make on the loadings $\beta_{i, 1}$ and $\mu_{i}$ guarantee that the explanatory power of the factors $F_{t, 1}$ and $v_{t}$ dominates that of the idiosyncratic error terms. The average squared loading on the factor $F_{2, t}$, however, converges to zero at the rate $1 / T$; if $N$ and $T$ increase proportionally, this will lead to factor $F_{2, t}$ having explanatory power comparable to that of the idiosyncratic errors. One characteristic of a weak factor is the following: 
if it is not observed we can neither consistently estimate it via the method of principle components nor consistently detect it.

The loadings $\beta_{i, 2}$ are asymptotically of the same order of magnitude as $\beta_{i, 1}$ divided by $\sqrt{T}$. Assumption LOADINGS makes that the standard deviation of the first-step estimate $\widehat{\beta}_{i, 2}$ be of the same order of magnitude as $\beta_{i, 2}$ itself. As we show, this is enough to make the two-pass estimator of the risk premia $\lambda_{2}$ on the weak factor $F_{t, 2}$ inconsistent and to invalidate the classical confidence interval for the risk premia $\lambda_{1}$ on the strong factor $F_{t, 1}$. The modeling assumption that makes $\beta_{i, 2}$ drift to zero at the $\sqrt{T}$ rate is similar to assumptions Kleibergen (2009) makes.

It is also important that the assumption on loadings $\mu_{i}$ are such that the unobserved factor $v_{t}$ in the error terms is strong. This is consistent with the empirical observations in Kleibergen and Zhan (2015). This also guarantees that the presence of the factor structure plays an important role in the asymptotics of two-pass estimation.

Assumption ERRORS. Assumptions ERRORS are high-level assumptions, whose main goal is to allow for very flexible weak cross-sectional dependence among the idiosyncratic errors, as well as flexible conditional heteroscedasticity and dependence in higher-order moments of errors and factors. The random variables $\rho(s, t)$ stand for a (normalized) empirical analog of the error autocorrelation coefficient, $S_{t}$ is an empirical variance, and $W_{t}$ is a (normalized) weighted average error. These variables are normalized so that they are stochastically bounded when the errors are cross-sectionally i.i.d.

Serial independence of errors as stated in Assumption ERRORS(i) is consistent with the efficient market hypothesis and the unpredictability of asset returns, and is generally consistent with empirical evidence and tradition in the literature. This assumption may be weakened, though we do not pursue this in the current paper.

In order to clarify the content of Assumptions ERRORS and to show that our assumptions are more flexible than those typically made in the literature, first, we provide below a set of more restrictive primitive assumptions that are common in the literature and that guarantee the validity of Assumptions ERRORS. Secondly, we also provide an empirically relevant example not covered by the primitive assumptions but which satisfies our more general Assumptions ERRORS. 


\section{Assumptions ERRORS*}

(i) The factors $\left\{F_{t}, t=1, \ldots, T\right\}$ are independent from the errors $\left\{e_{i t}, i=1, \ldots, N, t=\right.$ $1, \ldots, T\}$; the error terms $e_{t}=\left(e_{1 t}, \ldots, e_{N t}\right)^{\prime}$ are serially independent and identically distributed for different $t$ with $E e_{i t}=0$ and $\sup _{i, t} E e_{i t}^{4}<\infty$.

(ii) Let $\mathcal{E}_{N, T}=E\left[e_{t} e_{t}^{\prime}\right]$ be the $N \times N$ cross-sectional covariance matrix. For some positive constants $a, c$ and $C$, we have $\lim _{N, T} \frac{1}{N} \operatorname{tr}\left(\mathcal{E}_{N, T}\right)=a$ and

$$
c<\liminf _{N, T \rightarrow \infty} \min \operatorname{eval}\left(\mathcal{E}_{N, T}\right)<\limsup _{N, T \rightarrow \infty} \max \operatorname{eval}\left(\mathcal{E}_{N, T}\right)<C .
$$

(iii) $E\left|\frac{1}{\sqrt{N}} \sum_{i=1}^{N}\left(e_{i t}^{2}-E e_{i t}^{2}\right)\right|^{2}<C$.

Lemma 1 Assumptions LOADINGS and ERRORS* imply Assumptions ERRORS.

The primitive Assumptions ERRORS* are very close to those standard in the literature. Numerous papers that establish inferences in factor models commonly assume that the set of factors $\left\{F_{t}, t=1, \ldots, T\right\}$ is independent from the set $\left\{e_{i t}, i=1, \ldots, N\right.$, $t=1, \ldots, T\}$, though cross-sectional dependence of errors is allowed; see, for example, Assumption D in Bai and Ng (2006). Many papers allow for both time-series and crosssectional error dependence. We exclude time-series dependence, which is justified by the efficient-market hypothesis in our application. Assumption ERRORS*(ii) is intended to impose only weak dependence cross-sectionally as expressed by the covariance matrix; similar assumptions appear in Onatski (2012) and in Bai and Ng (2006).

Our high-level Assumptions ERRORS are more general than the more standard primitive Assumptions ERRORS*. In particular, our assumptions allow for very flexible conditional heteroscedasticity in the error terms and time-varying cross-sectional dependence, which seems relevant when we consider observed factors that characterize market conditions like the momentum factor. Consider the following example.

Example 3. Assume that errors $e_{i t}$ have the following weak latent factor structure:

$$
e_{i t}=\pi_{i}^{\prime} w_{t}+\eta_{i t}
$$

where $\left(w_{t}, F_{t}\right)$ is stationary, $w_{t}$ is $k_{w} \times 1$, serially independent conditional on $\mathcal{F}$, with $E\left(w_{t} \mid \mathcal{F}\right)=0$ and $E\left(w_{t} w_{t}^{\prime}\right)=I_{k_{w}}$ (normalization). Assume $E\left[\left(\left\|F_{t}\right\|^{4}+1\right)\left(\left\|w_{t}\right\|^{4}+1\right)\right]<$ 
$\infty$. We assume that the loadings satisfy the condition $\sum_{i=1}^{N} \pi_{i} \pi_{i}^{\prime} \rightarrow \Gamma_{\pi}$ (the factors $w_{t}$ are weak), and $N^{-1 / 2} \sum_{i=1}^{N} \pi_{i} \gamma_{i}^{\prime} \rightarrow \Gamma_{\pi \gamma}$. Assume that the random variables $\eta_{i t}$ are independent both cross-sectionally and across time, are independent from $w_{t}$ and $F_{t}$, and have mean zero and finite fourth moments and variances $\sigma_{i}^{2}$ that are bounded above and are such that $N^{-1} \sum_{i=1}^{N} \sigma_{i}^{2} \rightarrow \sigma^{2}$. As proven in the Supplementary Appendix, this example satisfies Assumptions ERRORS.

An interesting feature of this example is that it allows the errors to be weakly crosssectionally dependent to the extent that they may possess a weak factor structure. Moreover, this factor structure may be closely related to the observed factors $F_{t}$, which causes the cross-sectional dependence among the errors $e_{i t}$ to change with the observed factors $F_{t}$ and allows a very flexible form of conditional heteroskedasicity. Indeed, the conditional cross-sectional covariance is

$$
E\left(e_{i t} e_{j t} \mid \mathcal{F}\right)=\pi_{i}^{\prime} E\left(w_{t} w_{t}^{\prime} \mid \mathcal{F}\right) \pi_{j}+\mathbb{I}_{\{i=j\}} \sigma_{i}^{2}
$$

Since we do not restrict $E\left(w_{t} w_{t}^{\prime} \mid \mathcal{F}\right)$ beyond the proper moment conditions, the strength of any cross-sectional dependence as well as error variances may change stochastically depending on the realizations of the observed factors. This flexibility is extremely relevant for observed factors such as the momentum. For example, one may consider $w_{t}=\varsigma_{t} g\left(F_{t}, F_{t-1}, \ldots\right)$, where $\varsigma_{t} \sim N(0,1)$ is independent from all other variables; then for a proper choice of the function $g(\cdot)$ one may observe higher volatility and cross-sectional dependence of the idiosyncratic error for higher values of the observed factor $F_{t}$.

\section{Asymptotic properties of the two-pass procedure}

In this section we derive a result concerning the asymptotic properties of the classical two-pass procedure in a correctly-specified factor pricing model that may or may not include weak observed factors and may or may not have missing factors in the errors. Let us introduce the following notation: $\widetilde{F}_{t}=F_{t}-\frac{1}{T} \sum_{s=1}^{T} F_{s}$ and

$$
\widetilde{\lambda}=\lambda+\frac{1}{T} \sum_{t=1}^{T} F_{t}-E F_{t}, \quad u_{i}=\frac{1}{T} \sum_{t=1}^{T} \Sigma_{F}^{-1} \widetilde{F}_{t} e_{i t} .
$$


Random quantity $\tilde{\lambda}$ is also known as ex-post risk premia, it was introduced in Shanken (1992) and is the object of interest in Raponi, Robotti and Zaffaroni (2017) and Kim and Skoulakis (2018).

Now let us introduce two asymptotically important terms, the meaning and the names of which will be explained in the discussion following Theorem 1, The first term we refer to as "attenuation bias" is

$$
B^{A}=-\left(\sum_{i=1}^{N} \widehat{\beta}_{i} \widehat{\beta}_{i}^{\prime}\right)^{-1} \sum_{i=1}^{N} u_{i} u_{i}^{\prime} \widetilde{\lambda}
$$

while the second term we will call the "omitted variable bias" is

$$
B^{O V}=\left(\sum_{i=1}^{N} \widehat{\beta}_{i} \widehat{\beta}_{i}^{\prime}\right)^{-1} \sum_{i=1}^{N} \widehat{\beta}_{i} \frac{\mu_{i}^{\prime}}{\sqrt{T}}\left(\eta_{v, T}-\eta_{T}^{\prime} \tilde{\lambda}\right)
$$

These terms are not biases in an exact sense as they are random, but rather they are sample analogues of the expressions that are classically known as attenuation and omitted variable biases. Notice that both quantities are infeasible as they depend on unobserved errors $e_{i t}$, unobserved factors $v_{t}$ and unknown parameters $\lambda$ and $\mu_{i}$. Both terms are $k_{F} \times 1$ vectors. Let $B_{1}^{A}$ and $B_{1}^{O V}$ denote $k_{1} \times 1$ sub-vectors containing the first $k_{1}$ components, while $B_{2}^{A}$ and $B_{2}^{O V}$ are $k_{2} \times 1$ sub-vectors of the last $k_{2}$ components of $B^{A}$ and $B^{O V}$, correspondingly. We also adopt the following notation: $\Gamma_{\beta_{2} \mu}$ is the $k_{2} \times k_{\mu}$ sub-block of matrix $\Gamma$ (defined in Assumption LOADINGS) corresponding to the limit of $N^{-1} \sum_{i=1}^{N} \sqrt{T} \beta_{i, 2} \mu_{i}$. Other sub-matrices are denoted similarly.

Theorem 1 Assume that the samples $\left\{r_{i t}, i=1, \ldots, N, t=1, \ldots, T\right\}$ and $\left\{F_{t}, t=1, \ldots, T\right\}$ come from a data-generating process that satisfies factor-pricing model (2) and assumptions FACTORS, LOADINGS and ERRORS. Let $\widehat{\lambda}_{T P}$ denote the estimate obtained via the conventional two-pass procedure. Let both $N$ and $T$ increase to infinity without restric- 
tions on relative rates. Then the following asymptotic statements hold simultaneously:

$$
\begin{aligned}
\left(\begin{array}{c}
\sqrt{T} B_{1}^{O V} \\
B_{2}^{O V}
\end{array}\right) & \Rightarrow\left(\left(I_{k_{\beta}} ; \widetilde{\eta}\right) \Gamma\left(I_{k_{\beta}} ; \widetilde{\eta}\right)^{\prime}+\mathcal{I}_{k_{2}} \Sigma_{u} \mathcal{I}_{k_{2}}\right)^{-1}\left(\Gamma_{\beta \mu}+\widetilde{\eta} \Gamma_{\mu \mu}\right)\left(\eta_{v}-\eta^{\prime} \lambda\right), \\
\left(\begin{array}{c}
\sqrt{T} B_{1}^{A} \\
B_{2}^{A}
\end{array}\right) & \Rightarrow-\left(\left(I_{k_{\beta}} ; \widetilde{\eta}\right) \Gamma\left(I_{k_{\beta}} ; \widetilde{\eta}\right)^{\prime}+\mathcal{I}_{k_{2}} \Sigma_{u} \mathcal{I}_{k_{2}}\right)^{-1} \mathcal{I}_{k_{2}} \Sigma_{u} \lambda, \\
\sqrt{T}(\widetilde{\lambda}-\lambda) & \Rightarrow N\left(0, \Omega_{F}\right),
\end{aligned}
$$

and

$$
\left(\begin{array}{c}
\sqrt{N T}\left(\widehat{\lambda}_{T P, 1}-\widetilde{\lambda}_{1}-B_{1}^{A}-B_{1}^{O V}\right) \\
\sqrt{N}\left(\widehat{\lambda}_{T P, 2}-\widetilde{\lambda}_{2}-B_{2}^{A}-B_{2}^{O V}\right)
\end{array}\right)=O_{p}(1)
$$

where $\Sigma_{u}=\Sigma_{F}^{-1} \Sigma_{S F^{2}} \Sigma_{F}^{-1}, \mathcal{I}_{k_{2}}=\left(\begin{array}{cc}0_{k_{1}, k_{1}} & 0_{k_{1}, k_{2}} \\ 0_{k_{2}, k_{1}} & I_{k_{2}}\end{array}\right)$ is a $k_{F} \times k_{F}$ matrix, and $\tilde{\eta}=\mathcal{I}_{k_{2}} \eta$ is a $k_{F} \times k_{v}$ random matrix (with $\eta$ as in Assumption FACTORS).

Theorem 1 states the rates of convergence for different parts of the two-pass estimator. Notice that the theorem does not impose a relative rate of increase between $N$ and $T$ as long as both increase to infinity simultaneously. One observation is that the two-pass procedure cannot estimate $\lambda$ at a rate faster than $\sqrt{T}$ despite the fact that the dataset has NT observations of portfolio excess returns, and one could expect the $\sqrt{N T}$ rate. This arises from the fact that the correct specification (10) if averaged across time, gives

$$
\bar{r}_{i}=\widetilde{\lambda} \beta_{i}+\bar{\varepsilon}_{i}
$$

Thus, even if $\beta_{i}$ were known, the 'true' coefficient $\tilde{\lambda}$ in the only ideal regression we have (that is, regression of average return on $\beta_{i}$ ) differs from the parameter $\lambda$ we want to estimate, by the term $\frac{1}{T} \sum_{t=1}^{T} F_{t}-E F_{t}$, which, if multiplied by $\sqrt{T}$, is asymptotically zero mean gaussian with variance $\Omega_{F}$. Notice that if all observed factors $F_{t}$ are excess returns themselves and are assumed to be priced by the same pricing model, then the asset pricing theory provides an alternative way of estimating risk premia. Namely, in such a case $\lambda=E F_{t}$, and the alternative estimate is $\widehat{\lambda}=\frac{1}{T} \sum_{t=1}^{T} F_{t}=\tilde{\lambda}$. However, this estimate is not valid if factors themselves are not excess returns or are not priced by the same model. 
Notice also that if the limits of the normalized $B^{O V}$ and $B^{A}$ are non-zero, then these

terms (together with $\widetilde{\lambda}_{1}$ ) asymptotically dominate estimation. Below we consider three cases covered by Theorem 1. The first one is the case with no weak observed factors $\left(k_{2}=0\right)$. In this case the theorem delivers the validity of the two-pass procedure, namely, that the two-pass estimator is consistent and asymptotically mean-zero gaussian. For the other two (more empirically relevant) cases - one with weak observed factors but no missing factors, the other with weak observed factors and missing strong factors - the two-pass procedure fails.

\subsection{No weak observed factors}

Corollary 1 Assume that the samples $\left\{r_{i t}, i=1, \ldots, N, t=1, \ldots, T\right\}$ and $\left\{F_{t}, t=1, \ldots, T\right\}$ come from a data-generating process that satisfies factor-pricing model (2) and assumptions FACTORS, LOADINGS and ERRORS with $k_{2}=0$ (no weak observed factors). Then,

$$
\sqrt{T}\left(\widehat{\lambda}_{T P}-\lambda\right) \Rightarrow \Gamma_{\beta \beta}^{-1} \Gamma_{\beta \mu}\left(\eta_{v}-\eta^{\prime} \lambda\right)+\lim \sqrt{T}(\widetilde{\lambda}-\lambda)
$$

where the limit on the right hand side is asymptotically gaussian with mean zero. If in addition there are no strong missing factors in the errors (that is, $\mu_{i}=0$ ), then

$$
\sqrt{T}\left(\widehat{\lambda}_{T P}-\lambda\right)=\sqrt{T}(\widetilde{\lambda}-\lambda)+o_{p}(1) \Rightarrow N\left(0, \Omega_{F}\right) .
$$

This is a positive statement about the two-pass procedure, which claims that if all observed factors are strong, then the two-pass procedure is $\sqrt{T}$-consistent and provides an asymptotically mean-zero gaussian estimate for the risk premia when both $N, T \rightarrow \infty$. If the error terms have a strong factor structure it does not lead to a bias, but may increase the asymptotic variance. If no strong missing factor structure is present in the error terms, then the two-pass procedure is asymptotically equivalent to the infeasible estimate $\widetilde{\lambda}$ and will have asymptotic variance $\Omega_{F}$. 


\subsection{Weak observed factors but no strong missing factors}

Corollary 2 Assume that the samples $\left\{r_{i t}, i=1, \ldots, N, t=1, \ldots, T\right\}$ and $\left\{F_{t}, t=1, \ldots, T\right\}$ come from a data-generating process that satisfies factor-pricing model (2) and assumptions FACTORS, LOADINGS and ERRORS with $k_{2} \geq 1$ (there are weak observed factors) and $k_{v}=0$ (no missing factor structure in errors). Then the following asymptotic statements hold jointly:

$$
\begin{aligned}
\sqrt{T}\left(\widehat{\lambda}_{T P, 1}-\lambda_{1}\right) & =\sqrt{T}\left(\widetilde{\lambda}_{1}-\lambda_{1}\right)+\sqrt{T} B_{1}^{A}+o_{p}(1) \\
\widehat{\lambda}_{T P, 2}-\lambda_{2} & =B_{2}^{A}+o_{p}(1)
\end{aligned}
$$

where

$$
\left(\begin{array}{c}
\sqrt{T} B_{1}^{A} \\
B_{2}^{A}
\end{array}\right) \rightarrow^{p}-\left(\Gamma+\mathcal{I}_{k_{2}} \Sigma_{u} \mathcal{I}_{k_{2}}\right)^{-1} \mathcal{I}_{k_{2}} \Sigma_{u} \lambda
$$

In the case when some of the observed factors have relatively small loadings (weak observed factors) the two-pass estimator will deviate from the classical case even if the idiosyncratic errors are not strongly correlated. The limit in equation (4) is a non-random, non-zero vector, and thus characterizes the asymptotic bias. The two-pass estimate $\widehat{\lambda}_{T P, 2}$ of the risk premia on weak factors $F_{t, 2}$ is inconsistent and converges in probability to an incorrect value. The two-pass estimate $\widehat{\lambda}_{T P, 1}$ of risk premia on strong factors $F_{t, 1}$ is $\sqrt{T}$-consistent, but has a bias of order $\frac{1}{\sqrt{T}}$, the same order of magnitude as the standard deviation of its asymptotic distribution. This leads to invalid inferences on the risk premia.

The result of Corollary 2 can be explained in terms of classical error-in-variables bias. The first-pass estimate $\widehat{\beta}_{i}$ of risk exposure coefficients $\beta_{i}$ contains estimation errors which are stochastically of order $O_{p}(1 / \sqrt{T})$ each:

$$
\widehat{\beta}_{i}=\left(\sum_{t=1}^{T} \widetilde{F}_{t} \widetilde{F}_{t}^{\prime}\right)^{-1} \sum_{t=1}^{T} \widetilde{F}_{t} r_{i t}=\left(\beta_{i}+u_{i}\right)\left(1+o_{p}(1)\right),
$$

where the $o_{p}(1)$ term is related to the difference between $\Sigma_{F}=E\left[\left(F_{t}-E F_{t}\right)\left(F_{t}-E F_{t}\right)^{\prime}\right]$ and $T^{-1} \sum_{t} \widetilde{F}_{t} \widetilde{F}_{t}^{\prime}$. As a result, the second-pass regression encounters an error-in-variables problem. In the case of exposure to a strong observed factor, the estimation error in $\widehat{\beta}_{i, 1}$ is asymptotically negligible compared to the size of the coefficient $\beta_{i, 1}$ itself, and so this

estimation error does not jeopardize consistency. However, the estimation error in $\widehat{\beta}_{i, 2}$ 
is asymptotically of the same order of magnitude as the coefficient itself. The first-pass

estimation errors in $\widehat{\beta}_{i, 2}$ behave like a classical measurement error in the following sense: the imposed assumptions guarantee that the estimation errors $u_{i, 2}$ for different assets are asymptotically uncorrelated and that they are asymptotically uncorrelated with $\beta_{i}$ themselves in the sense that the sample correlation between $\beta_{i}$ and $u_{i}$ is asymptotically negligible. The bias we observe in Corollary 2 is a classical attenuation bias, with $\mathcal{I}_{k_{2}} \Sigma_{u} \mathcal{I}_{k_{2}}$ corresponding to the variance of the normalized measurement error $\sqrt{T} u_{i, 2}$.

Remark. A result similar to Corollary 2 can be derived under assumptions that $N$ is fixed while $T \rightarrow \infty$ and is due to Kleibergen (2009).

Note that if $\Gamma=\Gamma_{\beta \beta}$ is a block diagonal matrix with $\Gamma_{\beta_{1} \beta_{2}}=0_{k_{1}, k_{2}}$, the two-pass procedure inferences about $\lambda_{1}$ will not be disturbed; namely, $\widehat{\lambda}_{T P, 1}$ will be $\sqrt{T}$-consistent and will have an asymptotically mean-zero gaussian distribution. The block-diagonality assumption, however, is a very strong one: it requires that the values of $\beta_{i, 1}$ be unrelated to the values of $\beta_{i, 2}$ for the same asset, which is both implausible and not supported in applications. For example, the sample correlation coefficient between portfolios' betas that correspond to the market portfolio and the betas that correspond to the SMB (HML) portfolio in the Fama-French dataset are equal to 0.73 (0.47).

\subsection{Weak observed factors and strong missing factors}

Corollary 3 Assume that the sample $\left\{r_{i t}, i=1, \ldots, N, t=1, \ldots, T\right\}$ and $\left\{F_{t}, t=1, \ldots, T\right\}$ comes from a data-generating process that satisfies factor-pricing model (2) and assumptions FACTORS, LOADINGS and ERRORS with $k_{2} \geq 1$ (there are weak observed factors) and $k_{v} \geq 1$ (there is a missing factor structure in errors). Then the following asymptotic statements hold simultaneously:

$$
\begin{aligned}
\sqrt{T}\left(\widehat{\lambda}_{T P, 1}-\lambda_{1}\right) & =\sqrt{T}\left(\widetilde{\lambda}_{1}-\lambda_{1}\right)+\sqrt{T} B_{1}^{A}+\sqrt{T} B_{1}^{O V}+o_{p}(1), \\
\widehat{\lambda}_{T P, 2}-\lambda_{2} & =B_{2}^{A}+B_{2}^{O V}+o_{p}(1)
\end{aligned}
$$


where

$$
\begin{aligned}
&\left(\begin{array}{c}
\sqrt{T} B_{1}^{O V} \\
B_{2}^{O V}
\end{array}\right) \Rightarrow\left(\left(I_{k_{\beta}} ; \widetilde{\eta}\right) \Gamma\left(I_{k_{\beta}} ; \widetilde{\eta}\right)^{\prime}+\mathcal{I}_{k_{2}} \Sigma_{u} \mathcal{I}_{k_{2}}\right)^{-1}\left(\Gamma_{\beta \mu}+\widetilde{\eta} \Gamma_{\mu \mu}\right)\left(\eta_{v}-\eta^{\prime} \lambda\right), \\
&\left(\begin{array}{c}
\sqrt{T} B_{1}^{A} \\
B_{2}^{A}
\end{array}\right) \Rightarrow-\left(\left(I_{k_{\beta}} ; \widetilde{\eta}\right) \Gamma\left(I_{k_{\beta}} ; \widetilde{\eta}\right)^{\prime}+\mathcal{I}_{k_{2}} \Sigma_{u} \mathcal{I}_{k_{2}}\right)^{-1} \mathcal{I}_{k_{2}} \Sigma_{u} \lambda .
\end{aligned}
$$

The distributions on the right hand side are non-gaussian and are not centered at zero.

This result covers a more general case which, as we argued before, is empirically quite relevant. Here some observed pricing factors may have relatively small loadings (weak factors), while errors are highly cross-sectionally correlated to the extent that they have strong missing factor structures. The two-pass estimate $\widehat{\lambda}_{T P, 2}$ of the risk premia on weak factors $F_{t, 2}$ is inconsistent and, asymptotically, has a poorly-centered, non-standard distribution. The two-pass estimate $\hat{\lambda}_{T P, 1}$ of risk premia on strong factors $F_{t, 1}$ is $\sqrt{T}$ consistent, but this estimate has a bias of order $\frac{1}{\sqrt{T}}$ and an asymptotically non-standard distribution. This makes standard inferences (based on the usual $t$-statistics) invalid.

In the presence of a strong factor structure in the errors, first-pass estimates have the following form:

$$
\widehat{\beta}_{i}=\left(\sum_{t=1}^{T} \widetilde{F}_{t} \widetilde{F}_{t}^{\prime}\right)^{-1} \sum_{t=1}^{T} \widetilde{F}_{t} r_{i t}=\left(\beta_{i}+\frac{\eta_{T} \mu_{i}}{\sqrt{T}}+u_{i}\right)\left(1+o_{p}(1)\right),
$$

where $\eta_{T}=\frac{1}{\sqrt{T}} \sum_{t=1}^{T} \Sigma_{F}^{-1} \widetilde{F}_{t} v_{t}^{\prime} \Rightarrow \eta$. Again, for the strong observed factors, the estimation error in $\widehat{\beta}_{i, 1}$ turns out to be asymptotically negligible when compared to the sizes of risk exposures $\beta_{i, 1}$ themselves, while the estimation errors in $\widehat{\beta}_{i, 2}-$ which are now equal to $\eta_{T} \mu_{i} / \sqrt{T}+u_{i}$ - are of the size $O_{p}(1 / \sqrt{T})$, which is the same order of magnitude as the $\beta_{i, 2}$ 's themselves.

The estimation errors of $\widehat{\beta}_{i, 2}$ distort the asymptotics and invalidate classical inferences. However, unlike the case covered by Corollary 2 , the estimation errors in this setting do not behave like classical measurement errors in two respects. First, the estimation errors for different assets are correlated due to the presence of the common component $\eta_{T}$ in all of them. Second, unless $\mu_{i}$ is cross-sectionally uncorrelated with $\beta_{i}$ (so that $\Gamma_{\beta \mu}=0_{k_{F}, k_{v}}$ ), the estimation error will be correlated with its own regressor $\beta_{i}$. 
There is an additional issue classically known as omitted variable bias. Let us look at the second pass (normalized) 'ideal' regression, which we can obtain by time-averaging equation (2):

$$
\sqrt{T} \bar{r}_{i}=\sqrt{T} \widetilde{\lambda}^{\prime} \beta_{i}+\eta_{v, T}^{\prime} \mu_{i}+\sqrt{T} \bar{e}_{i}
$$

where $\eta_{v, T}=\frac{1}{\sqrt{T}} \sum_{t=1}^{T} v_{t} \Rightarrow \eta_{v} \sim N\left(0_{k_{v}, 1}, I_{k_{v}}\right)$. Here we introduced normalization $\sqrt{T}$ to make regression (6) more compatible with the classical OLS setup. The regression error terms $\sqrt{T} \bar{e}_{i}$ all have orders of magnitude of $O_{p}(1)$, zero means and finite variances. Even though in finite samples $\sqrt{T} \bar{e}_{i}$ may be weakly cross-sectionally dependent, Assumption ERRORS guarantees that they are asymptotically uncorrelated. Imagine for a moment that we know $\beta_{i}$ and $\mu_{i}$ for all assets. Then, regression (66) will take the form of a classic OLS regression, with regressors $\sqrt{T} \beta_{i, 2}$ and $\mu_{i}$ being of order of magnitude $O(1)$, in the sense expressed in Assumption LOADINGS, that in the classical regression setting would lead to a $\sqrt{N}$-consistent and asymptotically gaussian OLS estimator of the coefficients on them. The regressor $\sqrt{T} \beta_{i, 1}$ is, in contrast, of order $O(\sqrt{T})$ and carries a lot of information which, in the classical regression setting, leads to an OLS estimator of the coefficient $\lambda_{1}$ on this regressor that is both super-consistent and asymptotically centered gaussian. However, because $\mu_{i}$ is unobserved, it becomes a part of the error term in the second-pass regression, making error terms cross-sectionally correlated; see, for example, Andrews (2005) for a similar phenomenon. A more classical reference for this phenomenon is that of omitted variable bias: if $\Gamma_{\beta \mu} \neq 0_{k_{F}, k_{v}}$, then even if there were no first-pass estimation error and we knew $\beta_{i}$, running an OLS in a regression of $\sqrt{T} \bar{r}_{i}$ on $\sqrt{T} \beta_{i}$ would produce invalid results due to the omission of $\mu_{i}$.

One question that may arise is whether or not the omitted variable bias is large. The answer to this question is closely related to the size of the cross-sectional correlation between $\beta_{i}$ and $\mu_{i}$ as expressed in $\Gamma_{\beta \mu}$. Unfortunately, there is no reliable empirical evidence on this, as $\mu_{i}$ is unobserved and $\beta_{i}$ is poorly estimated and biased in the direction of $\mu_{i}$ (see equation (5) ). The problem with estimation of $\mu_{i}$ is that the estimator $\widehat{\lambda}_{T P, 2}$ is inconsistent, which makes the residuals from the two-pass procedure poor indicators of the true errors, and estimating $\mu_{i}$ via the principle components analysis on the residuals 
does not produce good estimates. However, even though direct empirical evidence on this matter is absent, we have two indirect arguments which suggest that one should expect a high rather than low correlation between $\beta_{i}$ and $\mu_{i}$. One argument is the empirical observation that for many well-known factor-pricing models the estimated betas for different factors are exceptionally highly correlated. Another argument is related to our theoretical example 1, where the missing factor structure originates as a result of mismeasuring the true risk factor, and the sample correlation between $\beta_{i}$ and $\mu_{i}$ equals 1 .

\section{Sample-split estimator of the risk premia}

\subsection{Idea of the proposed solution}

The case of no factor structure in the error terms. We begin by solving the easier case when no unobserved factor structure is present in the errors, while some observed factors are weak. In such a case the failure of the two-pass procedure can be labeled a classical measurement error-in-variables problem, which is often solved by finding a proper instrument. Apparently, it is relatively easy to find a valid instrument in our setting if one is willing to employ a sample-splitting technique.

Let us divide the set of time indexes $t=1, \ldots, T$ into two non-intersecting equal subsets $T_{1}$ and $T_{2}$. It is more natural to make $T_{1}$ the first half of the sample, and $T_{2}$ its second half. Let us run the first-step regression twice - separately on each sub-sample:

$$
\widehat{\beta}_{i}^{(j)}=\left(\sum_{t \in T_{j}} \widetilde{F}_{t}^{(j)} \widetilde{F}_{t}^{(j) \prime}\right)^{-1} \sum_{t \in T_{j}} \widetilde{F}_{t}^{(j)} r_{i t}=\left(\beta_{i}+u_{i}^{(j)}\right)\left(1+o_{p}(1)\right), \text { for } j=1,2,
$$

where $\widetilde{F}_{t}^{(j)}=F_{t}-\frac{1}{\left|T_{j}\right|} \sum_{t \in T_{j}} F_{t}, u_{i}^{(j)}=\frac{1}{\left|T_{j}\right|} \sum_{t \in T_{j}} \Sigma_{F}^{-1} \widetilde{F}_{t}^{(j)} e_{i t}$, and the $o_{p}(1)$ term is related to the difference between $\Sigma_{F}$ and $\frac{1}{\left|T_{j}\right|} \sum_{t \in T_{j}} \widetilde{F}_{t}^{(j)} \widetilde{F}_{t}^{(j) \prime}$.

The assumption ERRORS guarantees that the two sets of estimation uncertainty, $\left\{u_{i}^{(1)}, i=1, \ldots, N\right\}$ and $\left\{u_{i}^{(2)}, i=1, \ldots, N\right\}$, are independent conditionally on $\mathcal{F}$. In fact, the asymptotic independence of the two sets of errors will hold more generally if one makes stationarity assumptions and controls the decay of time-series dependence in errors $e_{i t}$, and the sub-samples are formed to be first and second halves of the sample. 
Given the observation about independence of estimation errors obtained from different sub-samples, one may use an estimate of $\beta_{i}$ from one sub-sample (for example, $\widehat{\beta}_{i}^{(1)}$ ) as a regressor while the other (in this example, $\widehat{\beta}_{i}^{(2)}$ ) as an instrument. This would represent a valid IV regression. Indeed, the second-step regression we run is:

$$
\bar{r}_{i}=\widetilde{\lambda}^{\prime} \widehat{\beta}_{i}^{(1)}+\left(\bar{e}_{i}-\widetilde{\lambda}^{\prime} u_{i}^{(1)}\right) .
$$

In this regression the regressor and the instrument are correlated since they both contain $\beta_{i}$, hence we get the relevance condition. The validity condition holds for two reasons: (i) the part of the second-step regression error $u_{i}^{(1)}$ is asymptotically uncorrelated with the instrument $\widehat{\beta}_{i}^{(2)}$; (ii) Assumption ERRORS guarantees that $\widehat{\beta}_{i}^{(2)}$ is asymptotically uncorrelated with $\bar{e}_{i}$. As we show below, this procedure restores consistency and standard inferences on the estimates of risk premia.

Similar ideas, such as sample splitting and jackknife-type estimators, have been previously employed in the literature on many weak instruments (e.g., Hansen, Hausman and Newey, 2008). In that literature the term "many instruments" is related to modeling the number of instruments as growing to infinity proportionally to the sample size or the concentration parameter, while the term "weak" appears due to a modeling assumption that makes the estimation error of the reduced-form coefficients be of the same order of magnitude as the coefficients themselves (so called local-to-zero asymptotics). This is parallel to the dimension asymptotics for a number of portfolios and the local-to-zero asymptotics for risk exposures of weak factors in our setup. In the many-weak-instrument setting, the regular TSLS estimator has a significant bias, and classical inferences are asymptotically invalid. That problem can also be interpreted as a classical measurement-error-in-variables problem for the second-stage regression, where the regression is run on the fitted values from the first-stage projection of the original regressor on the instruments. Some proposed solutions employ the second-stage instrumental variables regression where, for each observation, the regressor is obtained from a first-stage regression run on a sub-sample that does not include that observation, and the original instrument is still used as an instrument (see Angrist, Imbens and Krueger (1999) and Dufour and Jasiak (2001)). This forces the first-stage error in the projection to be uncorrelated with the instrument for this specific observation. Sample-splitting or leave-one-out type procedures restore consistency and 
classical inferences.

Raponi, Robotti and Zaffaroni (2017) and Kim and Skoulakis (2018) consider a similar phenomenon by assuming that $N \rightarrow \infty$ while $T$ is fixed. They show that in such a setting, the estimation error of the first stage is important and produces an inconsistent estimator of ex-post risk premia due to error-in-variable bias. These papers suggest correcting attenuation bias by directly estimating it. Those suggestions can be proved consistent in our asymptotic setting under slightly stronger Assumptions ERRORS. However, these estimation techniques would fail in the presence of missing factors in the error term.

The case of factor structure in the error terms. The model with unobserved factor structure has an additional problem - the presence of omitted (and unobserved) variable $\mu_{i}$ in regression (6). After examining formula (5) for the first-pass estimate we may notice that we can obtain a noisy proxy for $\mu_{i}$ if we take the difference between two estimates for the same $\beta_{i}$ obtained from different sub-samples. Indeed, consider two nonintersecting subsets of time indexes, $T_{1}$ and $T_{2}$, and assume they have the same number, say $\tau$, of time indexes. Then

$$
\widehat{\beta}_{i}^{(1)}-\widehat{\beta}_{i}^{(2)}=\frac{\eta_{\tau}^{(1)}-\eta_{\tau}^{(2)}}{\sqrt{\tau}} \mu_{i}+\left(u_{i}^{(1)}-u_{i}^{(2)}\right) .
$$

Notice that both the coefficient on $\mu_{i}$ and the noise term $u_{i}^{(1)}-u_{i}^{(2)}$ are of the same order of magnitude $O_{p}(1 / \sqrt{\tau})$. This means that neither the signal dominates the noise - and thus we need a correction to account for the noise, - nor the noise dominates the signal, and thus the proxy is not useless.

Assume that $k_{v} \leq k_{F}$, which implies that we have a larger number of proxies than needed, and we have a choice among them. Now we assume that we have a fixed and full-rank $k_{v} \times k_{F}$ matrix $A$, and use $A\left(\widehat{\beta}_{i}^{(1)}-\widehat{\beta}_{i}^{(2)}\right)$ as the proxy.

The idea is to regress the average return $\bar{r}_{i}$ on $\widehat{\beta}_{i}^{(1)}$ and $A\left(\widehat{\beta}_{i}^{(1)}-\widehat{\beta}_{i}^{(2)}\right)$ instead of on unobserved $\beta_{i}$ and $\mu_{i}$. This solves the omitted-variables part of the problem, but the error-in-variables issue still remains. That problem we solve via instrumental variables upon additional sample splitting. The ultimate idea goes as follows: split the sample into four equal sub-samples along the time dimension; calculate the first-pass estimates of risk exposures for all four sub-samples; run an instrumental variables regression using $\widehat{\beta}_{i}^{(1)}$ and 
$A\left(\widehat{\beta}_{i}^{(1)}-\widehat{\beta}_{i}^{(2)}\right)$ as regressors and $\widehat{\beta}_{i}^{(3)}$ and $\left(\widehat{\beta}_{i}^{(3)}-\widehat{\beta}_{i}^{(4)}\right)$ as instruments.

One can re-write the two pass-procedure as a GMM moment condition, then the twopass will correspond to an IV estimator with many instruments, as described in Newey and Windmeijer (2009). The problem described in Corollary 3 and the solution proposed in section 5.1 do not fall within the Newey and Windmeijer (2009) framework. The main departure is that Newey and Windmeijer (2009) consider i.i.d. sampling, while the observations in our model are so highly dependent that they produce inconsistency in the estimator.

\subsection{Algorithm for constructing four-split estimator}

Let us divide the set of time indexes into four equal non-intersecting subsets $T_{j}, j=1, \ldots, 4$.

(1) For each asset $i$ and each subset $j$ run a time-series regression to estimate the coefficients of risk exposure:

$$
\widehat{\beta}_{i}^{(j)}=\left(\sum_{t \in T_{j}} \widetilde{F}_{t}^{(j)} \widetilde{F}_{t}^{(j) \prime}\right)^{-1} \sum_{t \in T_{j}} \widetilde{F}_{t}^{(j)} r_{i t} .
$$

(2) Run an IV regression of $\bar{r}_{i}=\frac{1}{T} \sum_{t=1}^{T} r_{i t}$ on regressors $x_{i}^{(1)}=\left(\widehat{\beta}_{i}^{(1) \prime},\left(\widehat{\beta}_{i}^{(1)}-\widehat{\beta}_{i}^{(2)}\right)^{\prime} A_{1}^{\prime}\right)^{\prime}$ with instruments $z_{i}^{(1)}=\left(\widehat{\beta}_{i}^{(3) \prime},\left(\widehat{\beta}_{i}^{(3)}-\widehat{\beta}_{i}^{(4)}\right)^{\prime}\right)^{\prime}$, where $A_{1}$ is a non-random $k_{v} \times k_{F}$ matrix of rank $k_{F}$. Let $\widehat{\lambda}^{(1)}$ be the TSLS estimate of the coefficient on regressor $\widehat{\beta}_{i}^{(1)}$.

(3) Repeat step (2) three more times exchanging indexes 1 to 4 circularly; that is, the $(2)^{n d}$ regression is an IV regression of $\bar{r}_{i}$ on regressors $x_{i}^{(2)}=\left(\widehat{\beta}_{i}^{(2) \prime},\left(\widehat{\beta}_{i}^{(2)}-\widehat{\beta}_{i}^{(3)}\right)^{\prime} A_{2}^{\prime}\right)^{\prime}$ with instruments $z_{i}^{(2)}=\left(\widehat{\beta}_{i}^{(4) \prime},\left(\widehat{\beta}_{i}^{(4)}-\widehat{\beta}_{i}^{(1)}\right)^{\prime}\right)^{\prime} ;$ denote the estimate as $\widehat{\lambda}^{(2)}$, etc.

(4) Obtain the four-split estimate as $\widehat{\lambda}_{4 S}=\frac{1}{4} \sum_{j=1}^{4} \widehat{\lambda}^{(j)}$.

(5) In order to compute an estimate of the covariance matrix for $\widehat{\lambda}_{4 S}$, denote by $X^{(j)}$ the $N \times k$ matrix of stacked regressors used in the $(j)^{t h} \mathrm{IV}$ regression, and by $Z^{(j)}$ the $N \times k_{z}$ matrix of instruments from this regression (here $k_{z}=2 k_{F}$ and $k=k_{F}+k_{v}$ ). 
Let $P_{Z}=Z\left(Z^{\prime} Z\right)^{-1} Z^{\prime}$, calculate the following matrices:

$$
\widehat{\Sigma}_{0}=\frac{1}{N} \sum_{i=1}^{N}\left(\begin{array}{c}
\widetilde{z}_{i}^{(1)} \widehat{\epsilon}_{i}^{(1)} \\
\ldots \\
\widetilde{z}_{i}^{(4)} \widehat{\epsilon}_{i}^{(4)}
\end{array}\right)\left(\begin{array}{c}
\widetilde{z}_{i}^{(1)} \widehat{\epsilon}_{i}^{(1)} \\
\ldots \\
\widetilde{z}_{i}^{(4)} \widehat{\epsilon}_{i}^{(4)}
\end{array}\right)^{\prime}, \quad G=\left(\begin{array}{cccc}
G_{1} & 0_{k, k} & 0_{k, k} & 0_{k, k} \\
0_{k, k} & G_{2} & 0_{k, k} & 0_{k, k} \\
0_{k, k} & 0_{k, k} & G_{3} & 0_{k, k} \\
0_{k, k} & 0_{k, k} & 0_{k, k} & G_{4}
\end{array}\right)
$$

where $\widehat{\epsilon}_{i}^{(j)}$ is $i^{t h}$ residual from the $(j)^{t h}$ IV regression, $\widetilde{z}_{i}^{(j)}=X^{(j) \prime} Z^{(j)}\left(Z^{(j) \prime} Z^{(j)}\right)^{-1} z_{i}^{(j)}$, and $G_{j}=\frac{1}{N} X^{(j) \prime} P_{Z^{(j)}} X^{(j)}$. Denote $R=(1,1,1,1)^{\prime} \otimes\left(\begin{array}{c}\frac{1}{4} I_{k_{F}} \\ 0_{k_{v}, k_{F}}\end{array}\right)$, a $4 k \times k_{F}$ matrix. Then,

$$
\widehat{\Sigma}_{4 S}=\frac{1}{N} R^{\prime} G^{-1} \widehat{\Sigma}_{0} G^{-1} R+\frac{1}{T} \widehat{\Omega}_{F}
$$

where $\widehat{\Omega}_{F}$ is a consistent estimator of the long-run variance of $F_{t}$.

\subsection{Consistency of the four-split estimator}

Theorem 2 Assume that the samples $\left\{r_{i t}, i=1, \ldots, N, t=1, \ldots T\right\}$ and $\left\{F_{t}, t=1, \ldots, T\right\}$ come from a data-generating process that satisfies factor pricing model (2) and assumptions FACTORS, LOADINGS and ERRORS. Let both $N$ and $T$ increase to infinity, then

$$
\begin{gathered}
\sqrt{T}\left(\widehat{\lambda}_{4 S, 1}-\lambda_{1}\right)=\sqrt{T}\left(\widetilde{\lambda}_{1}-\lambda_{1}\right)+O_{p}(1 / \sqrt{N}) \Rightarrow N\left(0, \Omega_{F}\right) \\
\sqrt{\min \{N, T\}}\left(\widehat{\lambda}_{4 S, 2}-\lambda_{2}\right)=O_{p}(1) .
\end{gathered}
$$

Theorem 2 establishes the consistency rate for the four-split estimator $\widehat{\lambda}_{4 S}$ under exactly the same assumptions we showed the failure of the two-pass procedure. The four-split estimator for the risk premia on the strong observed factor is $\sqrt{T}$-consistent, asymptotically equivalent to $\widetilde{\lambda}_{1}$ and asymptotically gaussian, while the four-split estimate of the risk premia on the weak observed factor is consistent, and the rate of convergence depends on the relative size of $N$ and $T$. Theorem 2 shows that the four-split estimator has superior asymptotic properties in comparison to the classical two-pass procedure.

Remark. If we consider an asymptotic setting where $N \rightarrow \infty$, while $T$ is fixed, we can prove that under slightly stronger assumptions on the error terms, the four-split estimate 
is consistent for ex-post risk premia $\tilde{\lambda}$, and the $t$-statistics are asymptotically gaussian. The distinction between ex-post and ex-ante risk premia is discussed in Shanken (1992) as well as in Raponi, Robotti and Zaffaroni (2017) and in Kim and Skoulakis (2018). We need to strengthen the cross-sectional dependence assumptions on the error terms to the extent that the Law of Large Numbers and Cental Limit Theorems hold when summation is done over the cross-sectional index only. Assumptions in Raponi, Robotti and Zaffaroni (2017) and Kim and Skoulakis (2018) are of this type. The assumptions in Theorem 2 are somewhat weaker than needed for fixed $N$ setting as getting limit theorems when both summation indexes increase to infinity is easier, especially given the over-time independence of error-terms.

Remark. This papers considers unconditional pricing models only, but the estimation procedure can be adapted to work in some conditional pricing applications as well. In conditional pricing models we have either exposure coefficients $\beta_{i}$ 's or risk premia $\lambda$ (or both) that are not constant but change slowly over time. By using as a dependent variable the average of the excess returns over a narrow window of the most recent observations, by using only $\beta_{i}^{(4)}$ as the regressor on the IV stage, and by adjusting the size of the most recent subsample $T_{4}$ to guarantee that $\beta_{i}$ is nearly stable, we can end up with a valid estimate of the current risk premia. Developing the details of such estimation is left for future research.

Remark. One important assumption for the validity of our procedure is that we know the number of missing factors $k_{v}$. One may compose our estimator with a consistent selector of the number of factors as Onatski (2009), Bai and Ng (2002) or Gagliardini, Ossola and Scaillet (2016b) do.

\section{Inference procedures using four-split estimator}

Theorem 2 shows that the new four-split estimator is consistent but does not provide a basis for confidence set construction or testing. In order to make use of Theorem 2 the researcher must know which observed factors are strong, and with that knowledge $\mathrm{s} /$ he can construct a confidence set for the risk premia only on the strong observed fac- 
tor. Apparently, the stated assumptions are not strong enough to obtain the asymptotic distribution of the full four-split estimator. Below we formulate the needed additional high-level assumptions and establish a result about statistical inferences using the foursplit estimator. We also provide primitive assumptions that will guarantee the validity of the additional assumptions in examples.

For a set of vectors $a_{j}$, we denote by $\left(a_{j}\right)_{j=1}^{4}=\left(a_{1}^{\prime}, \ldots, a_{4}^{\prime}\right)^{\prime}$ a long vector consisting of the four vectors stacked upon each other; we denote by $\left(a_{j j^{*}}\right)_{j<j^{*}}$ the vectors $a_{j j^{*}}$ stacked together.

Assumption GAUSSIANITY Assume that the following convergence holds:

$$
\frac{1}{\sqrt{N}} \sum_{i=1}^{N}\left(\begin{array}{c}
\sqrt{T} \gamma_{i} \bar{e}_{i} \\
\left(\sqrt{T} \gamma_{i} u_{i}^{(j)}\right)_{j=1}^{4} \\
\left(T \bar{e}_{i} u_{i}^{(j)}\right)_{j=1}^{4} \\
\left(T u_{i}^{(j)} u_{i}^{\left(j^{*}\right)}\right)_{j<j^{*}}
\end{array}\right)=\frac{1}{\sqrt{N}} \sum_{i=1}^{N} \xi_{i} \Rightarrow \xi=\left(\begin{array}{c}
\xi_{\gamma e} \\
\left(\xi_{\gamma j}\right)_{j=1}^{4} \\
\left(\xi_{e j}\right)_{j=1}^{4} \\
\left(\xi_{j, j^{*}}\right)_{j<j^{*}}
\end{array}\right)
$$

where $\xi$ is a gaussian vector with mean zero and covariance $\Sigma_{\xi}$.

Assumption COVARIANCE Assume that $\frac{1}{N} \sum_{i=1}^{N} \xi_{i} \xi_{i}^{\prime} \rightarrow^{p} \Sigma_{\xi}$, where $\xi_{i}$ and $\Sigma_{\xi}$ are defined in Assumption GAUSSIANITY.

The assumptions we maintained in the previous sections are enough to guarantee that $\frac{1}{\sqrt{N}} \sum_{i=1}^{N} \xi_{i}$ is $O_{p}(1)$. Assumption GAUSSIANITY establishes the asymptotic distribution of that quantity, while Assumption COVARIANCE allows one to construct valid standard errors. Below we provide sufficient conditions for the two new assumptions in the two leading examples discussed before: one where the observed factors are independent from the errors and the example of factor-driven conditional heteroskedasticity.

Lemma 2 Assume that Assumption ERROR* holds and additionally,

(i) $E\left\|F_{t}\right\|^{8}<\infty ; E\left\|\frac{1}{\left|T_{j}\right|} \sum_{t \in T_{j}} F_{t} F_{t}^{\prime}-\Sigma_{F}\right\| \rightarrow 0$;

(ii) $\max _{i}\left\|\gamma_{i}\right\|<C$;

(iii) $\frac{1}{N} \operatorname{tr}\left(\mathcal{E}_{N, T}^{2}\right) \rightarrow a_{2}$ and $\frac{1}{N} \gamma^{\prime} \mathcal{E}_{N, T} \gamma \rightarrow \Gamma_{\sigma}$, where $\Gamma_{\sigma}$ is a full rank matrix; 
(iv) $\frac{1}{N^{2}} \sum_{i_{1}=1}^{N} \sum_{i_{2}=1}^{N} \sum_{i_{3}=1}^{N} \sum_{i_{4}=1}^{N}\left|E e_{i_{1} t} e_{i_{2} t} e_{i_{3} t} e_{i_{4} t}\right|<C$;

then Assumption GAUSSIANITY holds. If in addition

$$
\left\|\mathcal{E}_{N, T}-\operatorname{dg}\left(\mathcal{E}_{N, T}\right)\right\| \rightarrow 0 \text { as } N, T \rightarrow \infty,
$$

then Assumption COVARIANCE holds as well.

Lemma 3 Assume we have a setting as in Example 3. Assume additionally that conditions (i) and (ii) of Lemma Q hold and the following is true:

(i) $E\left[\left(\left\|F_{t}\right\|^{8}+1\right)\left\|w_{t}\right\|^{8}\right]<\infty$;

(ii) $\frac{1}{N} \sum_{i=1}^{N} \sigma_{i}^{4} \rightarrow \mu_{4}$ and $\frac{1}{N} \sum_{i=1}^{N} \sigma_{i}^{2} \gamma_{i} \gamma_{i}^{\prime} \rightarrow \Gamma_{\sigma}$, where $\Gamma_{\sigma}$ is a full rank matrix.

Then Assumption GAUSSIANITY holds. If in addition $\Gamma_{\pi \gamma}=0$, then Assumption COVARIANCE holds as well.

Assumption GAUSSIANITY is a result of strengthening moment restrictions (condition (i) in both Lemmas), guaranteeing that the asymptotic covariance matrix is well defined and full rank (condition (iii) in Lemma 2 and condition (ii) in Lemma 3) and further restricting cross-sectional dependence (condition (iv) in Lemma 2).

From a theoretical perspective, the derivation of a proper Central Limit Theorem in a factor model setting with a relatively free cross-sectional dependence structure is a major endeavor for two reasons. The first difficulty here is that the quite unrestrictive structure of the cross-sectional dependence of idiosyncratic error terms $e_{i t}$ makes $\xi_{i}$ cross-sectionally dependent, though the correlation between $\xi_{i}$ and $\xi_{i^{*}}$ for $i \neq i^{*}$ converges to zero for large sample sizes. Without imposing further discipline on the structure of dependence, it is hard to obtain a Central Limit Theorem. Secondly, the components $\xi_{e j}$ and $\xi_{j, j^{*}}$ are quadratic forms in the initial errors. Here we use the asymptotic results established for exactly this setting in a separate paper by Anatolyev and Mikusheva (2018). There, we exploit time-series conditional independence of errors to obtain a Central Limit Theorem for cross-sectional sums.

Theorem 3 Assume that the samples $\left\{r_{i t}, i=1, \ldots, N, t=1, \ldots T\right\}$ and $\left\{F_{t}, t=1, \ldots, T\right\}$ come from a data-generating process that satisfies factor pricing model (2) and Assumptions FACTORS, LOADINGS, ERRORS and GAUSSIANITY as both $N$ and T increase 
to infinity. Then $\sqrt{\min \{N, T\}}\left(\widehat{\lambda}_{4 S, 2}-\lambda_{2}\right)$ weakly converges to a mixed gaussian distribution. If in addition Assumption COVARIANCE holds, then

$$
\widehat{\Sigma}_{4 S}^{-1 / 2}\left(\widehat{\lambda}_{4 S}-\lambda\right) \Rightarrow N\left(0, I_{k}\right)
$$

Theorem 3 suggests the use of $t$ - and Wald statistics for the construction of confidence sets and testing hypotheses about values of the risk premia. These inference procedures are standard and can be implemented using standard econometrics software.

From a theoretical perspective, however, the asymptotics of the four-split estimator are not fully standard. The asymptotic distribution of the four-split estimator is not gaussian but rather mixed gaussian, that is, the limit distribution of the four-split estimator can be written as a gaussian random vector with random variance. To understand the intuition, look at equation (6) and notice that the coefficient $\eta_{v, T}$ on the omitted variable $\mu_{i}$ is random, even asymptotically. This implies that the amount of information contained in the sample, which is used to correct for the omitted-variable problem, is random as well, and thus results in an asymptotically random covariance matrix. Theorem 3 shows that a properly constructed proxy for the asymptotic variance restores the asymptotic gaussianity of a multidimensional $t$-statistic even when the estimator itself is not asymptotically gaussian. A similar phenomenon has appeared before in the literature on estimation of co-integrating relations.

Another important aspect of Theorem 3 is that inferences or construction of a proxy for the variance do not assume knowledge of the number or identity of strong/weak factors. This is a desirable feature, as we do not have a procedure that can credibly differentiate between weak and strong factors.

As previously discussed, even though the main data set contains NT observations, the risk premia cannot be estimated at a rate better than $\sqrt{T}$. This can be seen from equation (3), as even if we know the true values of $\beta_{i}$ the regression of $\bar{r}_{i}$ on $\beta_{i}$ has a true coefficient equal to $\widetilde{\lambda}=\lambda+\bar{F}-E F_{t}$. This means that the uncertainty associated with the deviations of $\frac{1}{T} \sum_{t=1}^{T} F_{t}$ from $E F_{t}$ is unavoidable. This also justifies the presence of the long-run variance of factors, $\Omega_{F}$, in the variance estimate $\widehat{\Sigma}_{4 S}$. Theorem 2 also states that the difference between $\widehat{\lambda}_{4 S}$ and $\widetilde{\lambda}$ is of order $\frac{1}{\sqrt{N T}}$. From the proof of Theorem 3 we see that this difference is mixed gaussian, and the variance can be deduced from 
$\widehat{\Sigma}_{I V}$. Typically, $\tilde{\lambda}$ is infeasible. However, if all observed factors are portfolios themselves and are priced by the same model, then we have $\lambda=E F_{t}$. In such a case the literature suggests the use of an alternative feasible estimator $\widehat{\lambda}=\frac{1}{T} \sum_{t=1}^{T} F_{t}$, which in this case is equal to $\tilde{\lambda}$. Thus, in this special case we have two competing estimators for $\lambda$ and can create a test for model specification. In particular, the statistic compares the difference between $\widehat{\lambda}_{4 S}$ and $\tilde{\lambda}$ to zero. The proof of Theorem 3 shows that $\widehat{\lambda}_{4 S}-\tilde{\lambda}$ converges to zero at the rate $\sqrt{N T}$, is asymptotically mixed gaussian, and $\widehat{\Sigma}_{I V}$ is a proper proxy for the variance that delivers a $\chi^{2}$ asymptotic distribution to the corresponding Wald statistic.

\section{$7 \quad$ Simulation evidence}

The goal of this section is to explore the size of potential deficiency of the two-pass and superior performance of the four-split procedure in a setting close to a real-life application. We expect the largest effect to come from the omitted-variable bias due to missing factor structure. The size of the bias and the distortion of the t-test coverage depend on the size of the missing factor, the strength of the observed factors, and the correlation between observed and missing factors. We explore these relations below.

\subsection{Simulations using artificial data}

Empirical setting. We calibrate the data-generating process in our simulations to match the data set of the monthly returns on 100 Fama-French portfolios sorted by size and book-to-market and asset returns' relation with the 3 Fama-French factors (market, SmB, HmL). The data is taken from Kenneth French's web-site. We substitute missing values with zeros. The returns are value-weighted, the excess returns are calculated using onemonth Treasury bills. The sample sizes are $N=100$ and $T=504$.

First, we run principle components $(\mathrm{PC})$ on the panel of excess returns, call the first three PCs $G_{t}$ with their loadings $\gamma_{i}$, and the fourth main principle component $g_{t}$ with loadings $\phi_{i}$. We use the normalization $\sum_{t=1}^{T}\left(G_{t}^{\prime}, g_{t}\right)^{\prime}\left(G_{t}^{\prime}, g_{t}\right)=I_{4}$, making the variance of each factor $1 / T$ (all results are invariant to the normalization). We compute sample means of the loadings, $\mu_{\gamma}$ and $\mu_{\phi}$, and their sample variances, $V_{\gamma}$ and $v_{\phi}$. We compute 
the residuals $\varepsilon_{i t}$ left after four main PCs, and compute their sample variance $\sigma_{\varepsilon}^{2}$. In order to preserve the relation between PCs and Fama-French factors, we run a regression of $F_{t}$ on a constant and $G_{t}$, obtain intercepts $\eta_{0, F}$, slopes $\eta_{F}$ and residual variance matrix $\Sigma_{r e s}$.

The fraction of the total variation explained by the four main PCs is approximately $73 \%, 6 \%, 3 \%$ and $1 \%$. In order to have a proper comparison consistent with our theoretical results, we measure the strength of a given factor as the total variation in the data it produces, that is, its strength is measured as the sum of squared loading on that factor times the variance of the factor. For example, for the fourth principle component $g_{t}$, its strength is measured as $\frac{1}{T} \sum_{i=1}^{N} \phi_{i}^{2}$. The strengths of the four main PCs and Fama-French factors are reported in Table 1. These numbers can serve as a reference for the simulation results below.

Table 1: The strength of principle components and Fama-French factors in the Fama-French data set, as measured by the total variation produced in the excess returns.

\begin{tabular}{l|cccc}
\hline \hline & 1st PC & 2nd PC & 3rd PC & 4th PC \\
\hline Strength of principle components & 2816 & 239 & 113 & 50 \\
\hline \hline & market & SmB & HmL & \\
\hline Strength of Fama-French factors & 2263 & 482 & 230 & \\
\hline \hline
\end{tabular}

Simulation design. We simulate the data by following three steps. In the first step we are trying to match the relation of the principle components and Fama-French factors. We simulate $G_{t} \sim$ i.i.d.N $\left(0, I_{3} / T\right)$ and $\gamma_{i} \sim$ i.i.d.N $\left(\mu_{\gamma}, V_{\gamma}\right)$, and then construct the simulated 'observed factors' by $F_{t}=\eta_{0, F}+\eta_{F} G_{t}+w_{t}$, where $w_{t} \sim$ i.i.d.N $\left(0, \Sigma_{\text {res }}\right)$.

As a second step we introduce one more factor to the returns, part of which will represent a missing factor structure in the errors, by simulating $g_{t} \sim$ i.i.d.N $(0,1 / T)$ and $\phi_{i} \sim$ i.i.d. $\vartheta_{\phi} \cdot N\left(\mu_{\phi}, v_{\phi}\right)$. The parameter $\vartheta_{\phi}$ indexes the strength of the missing factor. Finally, we introduce one more observed factor, which we label as mom because we want to imitate the relation of the momentum factor to the PCs. The analogy stops here: we do not claim to mimic the true momentum factor. We simulate $m_{0}=\eta_{0, \text { mom }}+\eta_{\text {mom }} G_{t}+$ $u_{t}+v_{t}$, where $\eta_{0, m o m}$ and $\eta_{m o m}$ are coefficients from a regression of the momentum factor on the PCs in the data. The simulated error consists of two parts: $v_{t} \sim$ i.i.d.N $\left(0, \varphi \sigma_{m o m}^{2}\right)$ is uncorrelated with returns or other factors, while $u_{t} \sim$ i.i.d.N $\left(0,(1-\varphi) \sigma_{\text {mom }}^{2}\right)$ will appear 
as a part of excess returns and is the reason for using mom in the pricing of assets. Here $\sigma_{m o m}^{2}$ is the estimated variance of the residuals from a regression of mom on PCs. In all simulations, we set $\varphi=0.001$.

We generate loadings on $u_{t}$ according to $\delta_{i}=\frac{\alpha \phi_{i} / \sqrt{T}+\xi_{i}}{\sqrt{(1-\varphi) \sigma_{\text {mom }}^{2}}}$, where $\xi_{i} \sim N\left(0, \sigma_{\xi}^{2}\right)$, so that they are (imperfectly) correlated with the loadings on $g_{t}$. Here by increasing $\alpha$ we can increase correlation, and by increasing $\sigma_{\xi}^{2}$ we can increase the strength of mom.

For the third step we generate a cross-section of returns and impose a correct pricing model. We generate the de-meaned part of excess returns according to $r_{i t}^{*}=G_{t} \gamma_{i}+g_{t} \phi_{i}+$ $u_{t} \delta_{i}+\epsilon_{i t}$, where $\epsilon_{i t} \sim$ i.i.d. $N\left(0, \sigma_{\varepsilon}^{2}\right)$ is an idiosyncratic error. The implied true betas are $\beta_{i}=\operatorname{var}\left(\begin{array}{c}F_{t} \\ m o m_{t}\end{array}\right)^{-1} \operatorname{cov}\left(\left(\begin{array}{c}F_{t} \\ m o m_{t}\end{array}\right), r_{i t}^{*}\right)=\operatorname{var}\left(\begin{array}{c}F_{t} \\ m o m_{t}\end{array}\right)^{-1}\left(\begin{array}{c}\eta_{F} \gamma_{i} / T \\ \eta_{m o m} \gamma_{i} / T+(1-\varphi) \sigma_{m o m}^{2} \delta_{i}\end{array}\right)$

Correctly priced excess returns are obtained by adding risk premia: $r_{i t}=r_{i t}^{*}+\lambda \beta_{i}$, where we set $\lambda$ to be sample means of the Fama-French and momentum factors from the data.

Simulation results. In all simulation experiments, we read off the biases, 'absolute' biases, standard deviations of the estimates for momentum risk premium, as well as the actual rejection rates of the null that the risk premium on the momentum factor equals its true value using the $5 \%$ nominal critical values. As we saw in equation (6) the omitted bias depends on the random variable $\eta_{v}$, which in different realizations of factors may be positive or negative with equal probability and washes out in repeated draws of timeseries processes. The 'absolute' bias is actually a better characterization of the centrality of a distribution. It is an absolute value of the bias averaged across $R_{(i)}=100$ draws of simulated cross sections (such as $\gamma_{i}, \phi_{i}$, etc.) averaged across each of $R_{(t)}=100$ draws of time-series processes (such as $G_{t}, F_{t}$, mom $_{t}$, etc.). Thus the total number of simulations is $R_{(i, t)}=R_{(i)} R_{(t)}=10,000$.

In the first set of experiments, we explore the effect of a change in the strength of the missing factor. We set $\alpha=0.1$ and $\sigma_{\xi}^{2}=0.3$, and then vary $\vartheta_{\phi}$ from 0 to 5 , the value 0 meaning no missing factors, the value 1 corresponding to the strength of the fourth PC. Figure 1 shows the performance measures for the conventional two-pass and proposed 

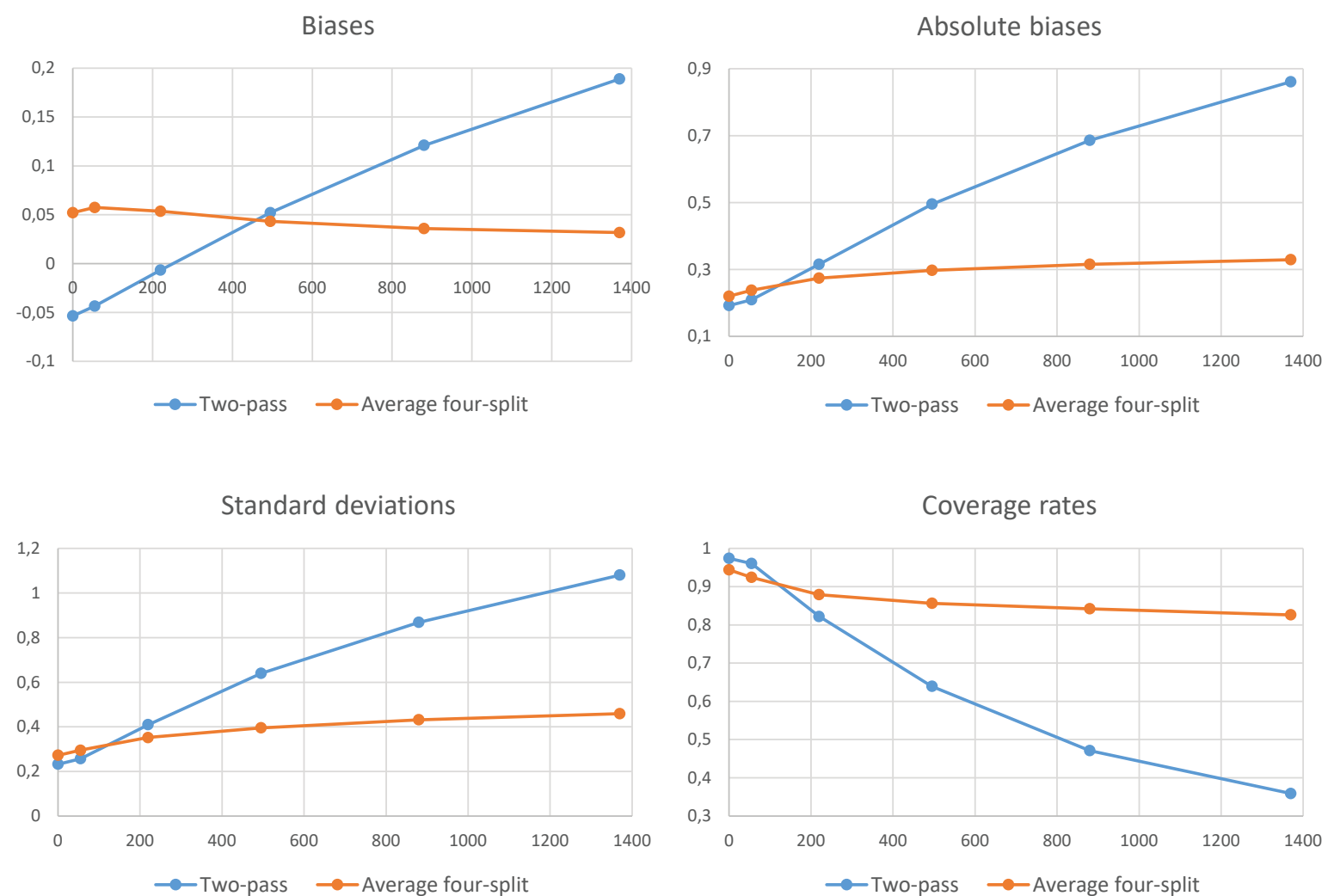

Figure 1: Simulated bias, absolute bias and standard deviation of the estimate and coverage of the $t$ statistics confidence for the risk premia on mom for two-pass and four-split methods. The strength of factor $g_{t}$ measured as total variation explained is on the horizontal axis. Number of simulations of the time series process $R_{(t)}=100$. For each time series draw, we simulate $R_{(i)}=100$ cross-section draws. Total number of simulations is 10,000 .

average four-split estimators, with the strength of the missing factor (as measured by the total variation explained by the factor, $\left.\frac{1}{T} \sum_{i=1}^{N} \phi_{i}^{2}\right)$ on the horizontal axis. From the bias panel, one can see that the two-pass estimator is unpredictable in 'biasedness' properties, and its bias can grow indefinitely, while the four-split estimator is much more stable, with the bias staying bounded while the missing factor grows in strength. More importantly, though, is that the absolute bias of the two-pass estimator grows fewfold larger than that of the four-split estimator. About the same happens to these estimators' standard deviations: the gap in variability grows fewfold as the missing factor grows in strength. Finally, the test size distortions may be smallest for the two-pass estimator in the absence of missing factors; they quickly become huge when the missing factor increases in importance. One can see that if the missing factor is of the size of $\mathrm{SmB}$ (see Table 1) 
one can easily get coverage around $60 \%$ instead of the declared $95 \%$.
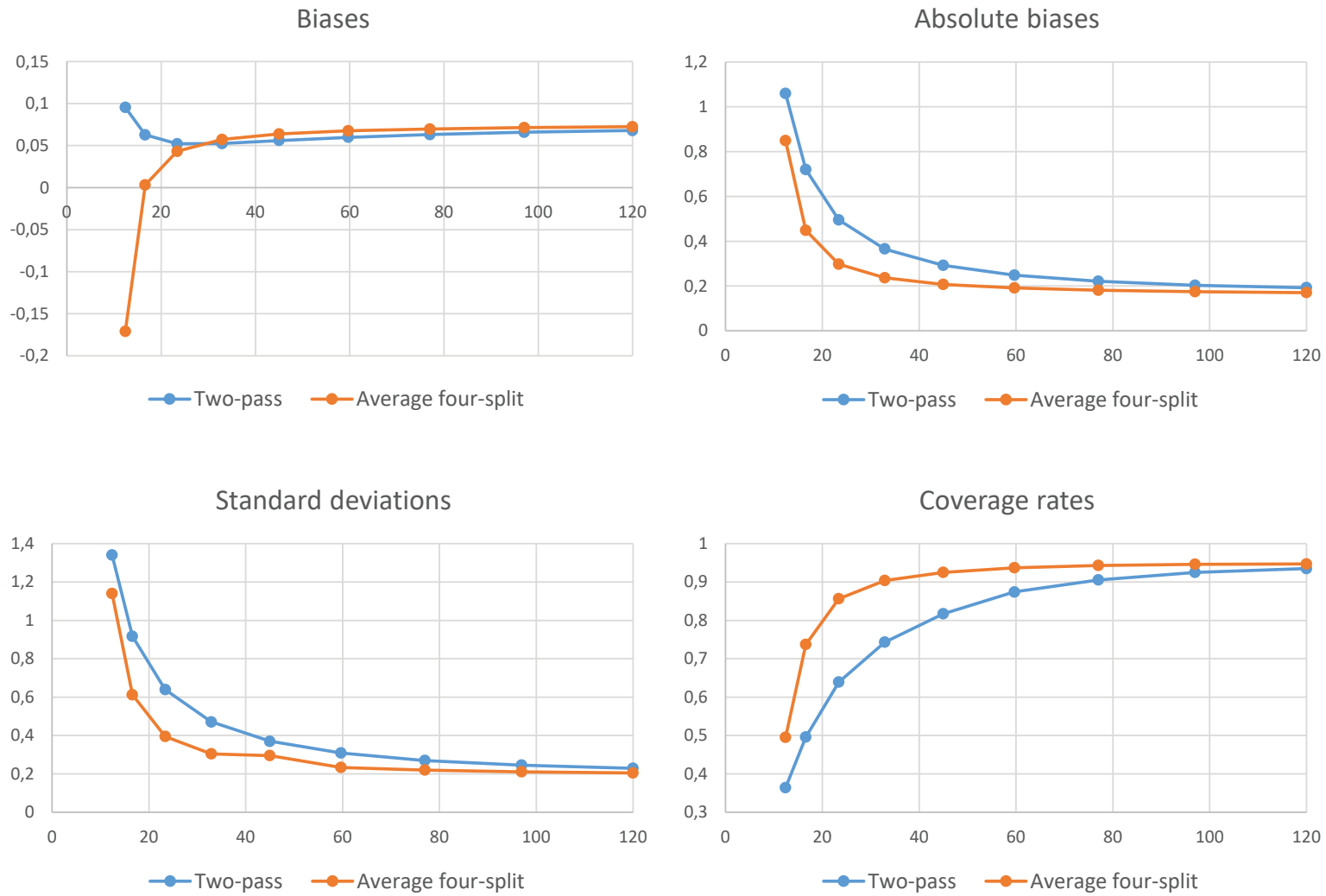

Figure 2: Simulated bias, absolute bias and standard deviation of the estimate and coverage of the $t$ statistics confidence for the risk premia on mom for two-pass and four-split methods. The strength of factor mom is on the horizontal axis. Number of simulations of the time series process $R_{(t)}=100$. For each time-series draw, we simulate $R_{(i)}=100$ cross-section draws. Total number of simulations is 10,000 .

In the second set of experiments, we set $\vartheta_{\phi}=3$ and $\alpha=0.1$, and then vary $\sigma_{\xi}^{2}$ from 0.1 to 0.9 with a step of 0.1 , which makes the strength of the missing factor (as measured by $\frac{1}{T} \sum_{i=1}^{N} \phi_{i}^{2}$ ) fixed at 495, and the strength of the momentum factor (as measured by $\left.\sum_{i=1}^{N} \beta_{i, 4}^{2} \frac{1}{T} \sum_{t=1}^{T}\left(\operatorname{mom}_{t}-\overline{m o m}\right)^{2}\right)$ increase from 12.4 to 120.0 . At the same time, the correlation between the missing and momentum factors (as measured by the sample correlation between $\phi_{i}$ and $\beta_{i, 4}$ ) decreases from 0.917 to 0.336 . Figure 2 shows the performance measures for the conventional two-pass and proposed average four-split estimators, with the strength of the momentum factor on the horizontal axis. While the ranking by the signed bias may not be predictable, there is clear dominance of the four-split estimator in terms of absolute bias, standard deviation, and actual rejection rates. The latter may be quite far from the nominal size when the momentum factor exhibits too much weakness, 
which in this case would be violation of Assumption LOADINGS.

\subsection{Size of the effect in an empirical application}

In this subsection we run the two-pass and four-split procedures on the data set of the monthly returns on 100 Fama-French portfolios sorted by size and book-to-market using 3 Fama-French factors (market, $\mathrm{SmB}, \mathrm{HmL}$ ) and the momentum factor as observed factors. The data are described previously. We estimate the model with the Fama-French factors only and then with all four factors. We report the results in Table 2, where for estimation of the long-run variance of observed factors we use the Newey-West estimator with 4 lags (though the results are not sensitive to a lag choice).

Table 2: Estimates of the risk premia on the Fama-French factors and the momentum factor and tests of specification, using monthly returns on 100 portfolios sorted by size and book-to-market. The covariance matrix is computed using the Newey-West estimator with 4 lags. The sample size is $T=504$.

\begin{tabular}{|c|c|c|c|c|c|c|}
\hline & Market & SMB & "HML & $\overline{\mathrm{MOM}}$ & "Wald & p-val \\
\hline average ex. return & $\begin{array}{c}0.527 \\
0.216 \\
\end{array}$ & $\begin{array}{l}0.187 \\
0.138\end{array}$ & $\begin{array}{c}0.401 \\
0.152\end{array}$ & $\begin{array}{l}0.708 \\
0.205 \\
\end{array}$ & & \\
\hline \multicolumn{7}{|c|}{ Model with 3 Fama-French factors } \\
\hline Two-pass & $\begin{array}{c}0.489 \\
0.218\end{array}$ & $\begin{array}{c}0.185 \\
0.146\end{array}$ & $\begin{array}{c}0.440 \\
0.165\end{array}$ & & 2.25 & 0.522 \\
\hline Four-split & $\begin{array}{c}0.218 \\
0.510 \\
0.219 \\
\end{array}$ & $\begin{array}{c}0.146 \\
0.167 \\
0.152 \\
\end{array}$ & $\begin{array}{c}0.105 \\
0.439 \\
0.165 \\
\end{array}$ & & 1.08 & 0.782 \\
\hline \multicolumn{7}{|c|}{ Model with 3 Fama-French factors and momentum } \\
\hline Two-pass & $\begin{array}{c}0.584 \\
0.218\end{array}$ & $\begin{array}{c}0.162 \\
0.145\end{array}$ & $\begin{array}{c}0.461 \\
0.161\end{array}$ & $\begin{array}{c}1.860 \\
0.385\end{array}$ & 21.99 & 0.000 \\
\hline Four-split & $\begin{array}{c}0.218 \\
0.534 \\
0.223 \\
\end{array}$ & $\begin{array}{c}0.145 \\
0.210 \\
0.159 \\
\end{array}$ & $\begin{array}{c}0.161 \\
0.436 \\
0.164 \\
\end{array}$ & $\begin{array}{c}0.385 \\
0.542 \\
0.637 \\
\end{array}$ & 3.96 & 0.411 \\
\hline
\end{tabular}

Since all the observed factors are tradable, we have an alternative (and the most efficient) estimator of the risk premia, $\widehat{\lambda}=\frac{1}{T} \sum_{t=1}^{T} F_{t}$, the average excess return, which is not available for non-tradable factors. This allows us to discuss the quality of different estimates relative to this benchmark. Also, having two valid estimates with different efficiency allows us to test for a correct specification of the linear pricing model (the Jtest). The specification test based on the Wald statistic is equal to the squared difference between the (two-pass or four-split) estimate and the average factor, weighted by an inverse of the difference in covariance matrices. The validity of such a test comes from the proof of Theorem 3. The results of the specification tests and the corresponding p-values appear in Table 2 . 
Notice that when we estimate the model with the three Fama-French factors only, both two-pass and four-split estimates are statistically indistinguishable from the average returns, so the Wald tests fail to reject the correct specification of the pricing model. Also, there is almost no cost in terms of efficiency of either estimate. When, however, we estimate the model with all four factors, the two-pass procedure produces a very high value for the risk premia on the momentum, and also falsely overstates the accuracy of that estimate to such an extent that the two-pass procedure strongly rejects the linear pricing model with four factors. This is an unfortunate outcome since the correct specification of the three-factor model implies a correct specification of the pricing model whenever one more factor is added. We attribute this strange behavior of the two-pass procedure to the momentum factor having only weak correlation with the returns. The four-split procedure, however, produces an estimate of the momentum risk premium close to the average excess return and accepts the correctness of the specification. The four-split estimate has much larger standard errors in comparison to the average excess returns, which is an implicit confirmation of the weakness of the momentum factor.

\section{References}

Anatolyev, S. (2012): "Inference in Regression Models with Many Regressors," Journal of Econometrics, 170(2), 368-382.

Anatolyev, S. And Mikusheva, A. (2018): "Limit Theorems for Factor Models," unpublished manuscript available at http://economics.mit.edu/files/15221 Andrews, D.W.K. (2005): "Cross-Section Regression with Common Shocks," Econometrica, 73(5), 1551-1585.

Angrist, J.D., G.W. Imbens And A. Krueger (1999): "Jackknife Instrumental Variables Estimation," Journal of Applied Econometrics, 14, 57-67.

BAI, J. AND NG, S. (2002): "Determining the Number of Factors in Approximate Factor Models," Econometrica, 70, 191-221.

BAI, J. AND S. NG (2006): "Confidence Intervals for Diffusion Index Forecast and Inference with Factor-Augmented Regressions", Econometrica, 74, 1133-1155. 
Bekker, P.A. (1994): "Alternative Approximations to the Distributions of Instrumental Variable Estimators," Econometrica, 62, 657-681.

Bryzgalova S. (2016): "Spurious Factors in Linear Asset Pricing Models", manuscript, Stanford Graduate School of Business.

Burnside, C. (2016): "Identification and Inference in Linear Stochastic Discount Factor Models with Excess Returns," Journal of Financial Econometrics, 14(2), 295-330.

Cochrane, J. (2001): Asset Pricing, Princeton University Press.

Dufour, J.M. And Jasiak, J. (2001): "Finite Sample Limited Information Inference Methods for Structural Equations and Models With Generated Regressors," International Economic Review, 42, 815-844.

FAma, E.F. And French, K.R. (1993): "Common Risk Factors in the Returns on Stocks and Bonds," Journal of Financial Economics, 33(1), 3-56.

FAmA, E.F. And MaCBeth, J. (1973): "Risk, Return and Equilibrium: Empirical Tests," Journal of Political Economy, 81, 607-636.

Feng, G., Giglio, S. And Xiu, D. (2019): "Taming the Factor Zoo: A Test of New Factors," NBER Working Paper No. 25481.

Gagliardini, P., Ossola, E. And Scaillet, O. (2016a) "Time-Varying Risk Premium in Large Cross-Sectional Equity Data Sets," Econometrica, 86, 985-1046.

Gagliardini, P., Ossola, E. And Scaillet, O. (2016b) "A Diagnostic Criterion for Approximate Factor Structure," Swiss Finance Institute Research Paper, 16-51.

Giglio, S. And Xiu, D. (2017): "Inference on Risk Premia in the Presence of Omitted Factors," NBER Working Paper No. 23527.

Gospodinov, N., Kan, R. And Robotti, C. (2017): "Spurious Inference in ReducedRank Asset-Pricing Models," Econometrica, 85(5), 1613-1628.

Hansen, C., Hausman, J. and Newey, W.K. (2008): "Estimation with Many Instrumental Variables," Journal of Business 86 Economics Statistics, 26, 398-422.

Harvey, C.R., Liu, Y. And Zhu, H. (2016): “... and the Cross-Section of Expected Returns," Review of Financial Studies, 29(1), 5-68.

Heyde, C. And Brown, B. (1970): "On the Departure from Normality of a Certain Class of Martingales," Annals of Mathematical Statistics, 41(6), 2161-2165. 
Jagannathan, R. And Wang, Z. (1996) The Conditional CAPM and the Cross-Section of Expected Returns. Journal of Finance, 51(1), 3-53.

Jegadeesh, N., Noh, J., Pukthuanthong, K., Roll, R., And Wang, J. (2019): "Empirical Tests of Asset Pricing Models with Individual Assets: Resolving the Error-inVariables Bias in Risk Premium Estimation," Journal of Financial Economics, forthcoming.

Jegadeesh, N. And Titman, S. (1993): "Returns to Buying Winners and Selling Losers: Implications for Stock Market Efficiency," Journal of Finance, 48(1), 65-91.

DE Jong, P. (1987): "A Central Limit Theorem for Generalized Quadratic Forms," Probability Theory and Related Fields, 75, 261-277.

Kan, R. And Zhang, C. (1999): "Two-Pass Tests of Asset Pricing Models with Useless Factors," Journal of Finance, 54, 203-235.

Kim, S. And Skoulakis, G. (2018): "Ex-post risk premia estimation and asset pricing tests using large cross sections: The regression-calibration approach," Journal of Econometrics, 24, 159-188.

Kleibergen, F. (2009): "Tests of Risk Premia in Linear Factor Models," Journal of Econometrics, 149(2), 149-173.

Kleibergen, F. And Zhan, Z. (2015): "Unexplained Factors and Their Effects on Second Pass R-squared's," Journal of Econometrics, 189, 101-116.

Kozak, S., Nagel, S. And Santosh, S. (2018): "Shrinking the Cross-Section," Journal of Financial Economics, forthcoming.

Lettau, M. And Ludvigson, S. (2001): "Resurrecting the (C)CAPM: A Cross-Sectional Test When Risk Premia Are Time-Varying," Journal of Political Economy, 109(6), 12381287.

Lettau, M. And Pelger, M. (2018): "Estimating Latent Asset-Pricing Factors," NBER Working Paper No. 24618.

Lintner, J. (1965): "Security Prices, Risk, and Maximal Gains from Diversification," Journal of Finance, 20, 587-615.

Lewellen, J., Nagel, S., And Shanken, J. (2010): “A Skeptical Appraisal of Asset Pricing Tests," Journal of Financial Economics, 96, 175-194. 
Newey, W.K. And Windmeijer, F. (2009): "Generalized Method of Moments With Many Weak Moment Conditions," Econometrica, 77, 687-719.

Onatski, A. (2012): "Asymptotics of the Principal Components Estimator of Large Factor Models with Weakly Influential Factors," Journal of Econometrics, 168, 244-258.

PÁAStor, L. And Stambaugh, R.F. (2003): Liquidity risk and expected stock returns," Journal of Political Economy, 111(3), 642-685.

Raponi, V., Robotti, C., and Zaffaroni, P. (2017): "Testing Beta-Pricing Models Using Large Cross-Sections," Working paper, Yale University.

Shanken, J. (1992): "On the Estimation of Beta-Pricing Models," Review of Financial Studies, 5, 1-33.

Sharpe, W.F. (1964): "Capital Asset Prices: A Theory of Market Equilibrium under Conditions of Risk," Journal of Finance, 19, 425-442.

Staiger, D. And Stock, J.H. (1997): "Instrumental Variables Regression with Weak Instruments," Econometrica, 65(3), 557-586.

\section{Appendix A: proofs}

Note that Lemma S1 often referred to is contained in the Supplementary Appendix.

Proof of Theorem 1. Assumption FACTORS guarantees that $\sqrt{T}(\widetilde{\lambda}-\lambda) \Rightarrow N\left(0, \Omega_{F}\right)$. The first-pass (time series) regression yields equation (15), where we use Assumption FACTORS, and $o_{p}(1)$ appears from the difference between $\Sigma_{F}$ and $T^{-1} \sum_{t=1}^{T} \widetilde{F}_{t} \widetilde{F}_{t}^{\prime}$.

Denote $Q_{T}=\left(\begin{array}{cc}I_{k_{1}} & 0_{k_{1}, k_{2}} \\ 0_{k_{2}, k_{1}} & \sqrt{T} I_{k_{2}}\end{array}\right)$. Notice that $Q_{T} / \sqrt{T} \rightarrow \mathcal{I}_{k_{2}}$. Below we prove the following statement: as $N, T \rightarrow \infty$,

$$
\frac{1}{N} \sum_{i=1}^{N} Q_{T} \widehat{\beta}_{i} \widehat{\beta}_{i}^{\prime} Q_{T} \Rightarrow\left(I_{k_{\beta}} ; \widetilde{\eta}\right) \Gamma\left(I_{k_{\beta}} ; \widetilde{\eta}\right)^{\prime}+\mathcal{I}_{k_{2}} \Sigma_{u} \mathcal{I}_{k_{2}}
$$


Indeed,

$$
\begin{aligned}
\frac{1}{N} \sum_{i=1}^{N} Q_{T} \widehat{\beta}_{i} \widehat{\beta}_{i}^{\prime} Q_{T} & =\frac{1}{N} \sum_{i=1}^{N}\left(Q_{T} \beta_{i}+Q_{T} \frac{\eta_{T}}{\sqrt{T}} \mu_{i}+Q_{T} u_{i}\right)\left(Q_{T} \beta_{i}+Q_{T} \frac{\eta_{T}}{\sqrt{T}} \mu_{i}+Q_{T} u_{i}\right)^{\prime} \\
& =\frac{1}{N} \sum_{i=1}^{N}\left(\left(I_{k_{\beta}} ; \widetilde{\eta}_{T}\right) \gamma_{i}+Q_{T} u_{i}\right)\left(\left(I_{k_{\beta}} ; \widetilde{\eta}_{T}\right) \gamma_{i}+Q_{T} u_{i}\right)^{\prime}
\end{aligned}
$$

where $\widetilde{\eta}_{T}=Q_{T} \eta_{T} / \sqrt{T} \Rightarrow \mathcal{I}_{k_{2}} \eta=\widetilde{\eta}$ is $k_{F} \times k_{v}$ gaussian random matrix. Let us show that

$$
\frac{T}{N} \sum_{i=1}^{N} u_{i} u_{i}^{\prime} \rightarrow \Sigma_{u}
$$

Indeed, due to statement (4) of Lemma S1 we have that $\frac{1}{T N} \sum_{i=1}^{N} \sum_{t=1}^{T} \sum_{s=1, s \neq t}^{T} \widetilde{F}_{t} \widetilde{F}_{s}^{\prime} e_{i t} e_{i s}=$ $o_{p}(1)$. Thus,

$$
\begin{aligned}
\frac{T}{N} \sum_{i=1}^{N} u_{i} u_{i}^{\prime} & =\Sigma_{F}^{-1}\left(\frac{1}{T N} \sum_{i=1}^{N} \sum_{t=1}^{T} \sum_{s=1}^{T} \widetilde{F}_{t} \widetilde{F}_{s}^{\prime} e_{i t} e_{i s}\right) \Sigma_{F}^{-1} \\
& =\Sigma_{F}^{-1}\left(\frac{1}{T N} \sum_{i=1}^{N} \sum_{t=1}^{T} \widetilde{F}_{t} \widetilde{F}_{t}^{\prime} e_{i t}^{2}\right) \Sigma_{F}^{-1}+o_{p}(1) \rightarrow^{p} \Sigma_{u}
\end{aligned}
$$

where the last convergence comes from statement (3) of Lemma S1. Statement (5) of Lemma S1 implies

$$
\frac{\sqrt{T}}{N} \sum_{i=1}^{N} \gamma_{i} u_{i}^{\prime} \rightarrow^{p} 0_{k, k_{F}} .
$$

Combination of equations (8)-(10) and Assumption LOADINGS implies (77).

For the "attenuation bias,"

$$
\left(\begin{array}{c}
\sqrt{T} B_{1}^{A} \\
B_{2}^{A}
\end{array}\right)=Q_{T}^{-1} \sqrt{T} B^{A}=-\left(\frac{1}{N} Q_{T} \sum_{i=1}^{N} \widehat{\beta}_{i} \widehat{\beta}_{i}^{\prime} Q_{T}\right)^{-1} \frac{Q_{T}}{\sqrt{T}} \frac{T}{N} \sum_{i=1}^{N} u_{i} u_{i}^{\prime} \widetilde{\lambda}
$$

Combining equations (17), (9),$\widetilde{\lambda} \rightarrow^{p} \lambda$ and $Q_{T} / \sqrt{T} \rightarrow \mathcal{I}_{k_{2}}$, we arrive at

$$
\left(\begin{array}{c}
\sqrt{T} B_{1}^{A} \\
B_{2}^{A}
\end{array}\right) \Rightarrow-\left(\left(I_{k_{\beta}} ; \widetilde{\eta}\right) \Gamma\left(I_{k_{\beta}} ; \widetilde{\eta}\right)^{\prime}+\mathcal{I}_{k_{2}} \Sigma_{u} \mathcal{I}_{k_{2}}\right)^{-1} \mathcal{I}_{k_{2}} \Sigma_{u} \lambda
$$

For the "omitted variable bias,"

$$
\left(\begin{array}{c}
\sqrt{T} B_{1}^{O V} \\
B_{2}^{O V}
\end{array}\right)=Q_{T}^{-1} \sqrt{T} B^{O V}=\left(\frac{1}{N} \sum_{i=1}^{N} Q_{T} \widehat{\beta}_{i} \widehat{\beta}_{i}^{\prime} Q_{T}\right)^{-1} \frac{1}{N} \sum_{i=1}^{N} Q_{T} \widehat{\beta}_{i} \mu_{i}^{\prime}\left(\eta_{v, T}-\eta_{T}^{\prime} \widetilde{\lambda}\right) .
$$


Let us consider the following expression:

$$
\frac{1}{N} \sum_{i=1}^{N} Q_{T} \widehat{\beta}_{i} \mu_{i}^{\prime}=\frac{1}{N} \sum_{i=1}^{N}\left(Q_{T} \beta_{i}+Q_{T} \frac{\eta_{T} \mu_{i}}{\sqrt{T}}+Q_{T} u_{i}\right) \mu_{i}^{\prime} .
$$

By Assumption LOADINGS, $\frac{1}{N} \sum_{i=1}^{N} Q_{T} \beta_{i} \mu_{i}^{\prime} \rightarrow \Gamma_{\beta \mu}$ and $\frac{1}{N} \sum_{i=1}^{N} \mu_{i} \mu_{i}^{\prime} \rightarrow \Gamma_{\mu \mu}$, while $Q_{T} \eta_{T} / \sqrt{T} \Rightarrow \widetilde{\eta}$. The last term in equation (11) is $o_{P}(1)$ by statement (5) of Lemma S1. Thus,

$$
\frac{1}{N} \sum_{i=1}^{N} Q_{T} \widehat{\beta}_{i} \mu_{i}^{\prime} \Rightarrow \Gamma_{\beta \mu}+\widetilde{\eta} \Gamma_{\mu \mu}
$$

We also note that $\eta_{v, T}-\eta_{T}^{\prime} \widetilde{\lambda} \Rightarrow \eta_{v}-\eta^{\prime} \lambda$. This implies validity of the asymptotic statement about $B^{O V}$ contained in Theorem 1 .

For the remaining part, by time averaging equation (21) we get $\bar{r}_{i}=\beta_{i}^{\prime} \widetilde{\lambda}+\mu_{i}^{\prime} \frac{\eta_{v, T}}{\sqrt{T}}+\bar{e}_{i}$. Combining the last equation with equation (15), we obtain

$$
\bar{r}_{i}=\widehat{\beta}_{i}^{\prime} \widetilde{\lambda}-u_{i}^{\prime} \widetilde{\lambda}+\frac{\mu_{i}^{\prime}}{\sqrt{T}}\left(\eta_{v, T}-\eta_{T}^{\prime} \widetilde{\lambda}\right)+\bar{e}_{i}
$$

Thus, we arrive at

$$
\begin{aligned}
\widehat{\lambda}_{T P}-\widetilde{\lambda}-B^{A}-B^{O V} & =\left(\sum_{i=1}^{N} \widehat{\beta}_{i} \widehat{\beta}_{i}^{\prime}\right)^{-1}\left(-\sum_{i=1}^{N}\left(\widehat{\beta}_{i}-u_{i}\right) u_{i}^{\prime} \widetilde{\lambda}+\sum_{i=1}^{N} \widehat{\beta}_{i} \bar{e}_{i}\right) \\
& =\left(\sum_{i=1}^{N} \widehat{\beta}_{i} \widehat{\beta}_{i}^{\prime}\right)^{-1}\left(-\sum_{i=1}^{N}\left(\beta_{i}+\frac{\eta_{T} \mu_{i}}{\sqrt{T}}+o_{p}(1)\right) u_{i}^{\prime} \widetilde{\lambda}+\sum_{i=1}^{N} \widehat{\beta}_{i} \bar{e}_{i}\right),
\end{aligned}
$$

SO

$$
\begin{aligned}
& \sqrt{N T} Q_{T}^{-1}\left(\widehat{\lambda}_{T P}-\widetilde{\lambda}-B^{A}-B^{O V}\right) \\
& \quad=\left(\frac{1}{N} \sum_{i=1}^{N} Q_{T} \widehat{\beta}_{i} \widehat{\beta}_{i}^{\prime} Q_{T}\right)^{-1} \sqrt{\frac{T}{N}}\left(-\sum_{i=1}^{N} Q_{T}\left(\beta_{i}+\frac{\eta_{T} \mu_{i}}{\sqrt{T}}\right) u_{i}^{\prime} \widetilde{\lambda}+\sum_{i=1}^{N} Q_{T} \widehat{\beta}_{i} \bar{e}_{i}\right) .
\end{aligned}
$$

Let us prove that the numerator is asymptotically $O_{p}(1)$ :

$$
\begin{aligned}
& \sqrt{\frac{T}{N}}\left(-\sum_{i=1}^{N} Q_{T}\left(\beta_{i}+\frac{\eta_{T} \mu_{i}}{\sqrt{T}}\right) u_{i}^{\prime} \widetilde{\lambda}+\sum_{i=1}^{N} Q_{T} \widehat{\beta}_{i} \bar{e}_{i}\right) \\
& \quad=\left(I_{k_{\beta}} ; \widetilde{\eta}_{T}\right) \sqrt{\frac{T}{N}} \sum_{i=1}^{N} \gamma_{i}\left(\bar{e}_{i}-u_{i}^{\prime} \tilde{\lambda}\right)+\sqrt{\frac{T}{N}} \sum_{i=1}^{N} Q_{T} u_{i} \bar{e}_{i}+O_{p}(1) .
\end{aligned}
$$

By statement $(5)$ of Lemma S1, we have $\sqrt{\frac{T}{N}} \sum_{i} \gamma_{i} \bar{e}_{i}=O_{p}(1)$ and $\sqrt{\frac{T}{N}} \sum_{i} \gamma_{i} u_{i}^{\prime}=O_{p}(1)$, which makes the first summand in equation (12) $O_{p}(1)$. Consider the second term in 
equation (12) and recall that $Q_{T} / \sqrt{T}=O(1)$ :

$$
\begin{aligned}
\sqrt{\frac{T}{N}} \sum_{i=1}^{N} Q_{T} u_{i} \bar{e}_{i} & =\frac{Q_{T}}{\sqrt{T}} \Sigma_{F}^{-1} \frac{1}{\sqrt{N} T} \sum_{i=1}^{N} \sum_{t=1}^{T} \sum_{s=1}^{T} \widetilde{F}_{s} e_{i s} e_{i t} \\
& =\frac{Q_{T}}{\sqrt{T}} \Sigma_{F}^{-1} \frac{1}{\sqrt{N} T} \sum_{i=1}^{N} \sum_{t=1}^{T} \sum_{s \neq t} \widetilde{F}_{s} e_{i s} e_{i t}+\frac{Q_{T}}{\sqrt{T}} \Sigma_{F}^{-1} \frac{\sqrt{N}}{T} \sum_{t=1}^{T} \widetilde{F}_{t} S_{t} .
\end{aligned}
$$

The first term is $O_{p}(1)$ by statement (4) of Lemma $\mathrm{S} 1$, while the second term is $O_{p}(1)$ by Assumption ERRORS(iii). This ends the proof of Theorem 1 .

Proof of Corollary 2. When we have weak included factors $\left(k_{2} \geq 1\right)$ but no strong excluded factors $\left(k_{v}=0\right)$, the expression for the omitted variables bias $\left(\mu_{i}=0\right)$ is exactly equal to zero: $B^{O V}=0$. In this case, $v_{t}=0$ and hence $\eta_{T}=0$, as well as $\eta=0$ and $\widetilde{\eta}=0$. That gives the expression (41) for the limit of the attenuation bias.

Proof of Corollary 1. If all observed factors are strong, then there is no second component to the risk premia, i.e., $k_{2}=0$ and $\lambda=\lambda_{1}$. We also have $\mathcal{I}_{k_{2}}=0_{k_{F}, k_{F}}$ and $\widetilde{\eta}=0$. When applied to the result of Theorem 1, we obtain that $\sqrt{T} B^{A} \rightarrow^{p} 0$ and

$$
\sqrt{T} B^{O V} \Rightarrow\left(\Gamma_{\beta \beta}\right)^{-1} \Gamma_{\beta \mu}\left(\eta_{v}-\eta^{\prime} \lambda\right)
$$

which is a zero mean gaussian limit. Thus, in this case we have

$$
\sqrt{T}(\widehat{\lambda}-\lambda)=\sqrt{T}(\widetilde{\lambda}-\lambda)+\sqrt{T} B^{O V}+o_{p}(1) .
$$

Finally, if in addition to $k_{2}=0$ we also have $k_{v}=0$ (no missing factor structure), then $B^{O V}=0$ exactly.

Proof of Theorem 2. We first discuss the asymptotics of just one IV regression described on step (2), then this argument will be repeated for the other three IV regressions from step (2) of the algorithm. Denote $\tau=\left\lfloor\frac{T}{4}\right\rfloor=\left|T_{j}\right|$.

The time-series regression on a sub-sample $j$ gives us that

$$
\widehat{\beta}_{i}^{(j)}=\left(\beta_{i}+u_{i}^{(j)}+\frac{\eta_{j, T} \mu_{i}}{\sqrt{\tau}}\right)\left(1+o_{p}(1)\right),
$$

where $\eta_{j, T}=\frac{1}{\sqrt{\tau}} \sum_{t \in T_{j}} \Sigma_{F}^{-1} \widetilde{F}_{t}^{(j)} v_{t}^{\prime} \Rightarrow \eta_{j}, \eta_{j}$ is random $k_{F} \times k_{v}$ matrix with the distribution $\operatorname{vec}\left(\eta_{j}\right) \sim N\left(0_{k_{F} k_{v}, 1}, \Omega_{v F}\right)$, and the $o_{p}(1)$ term is related to the difference between $\Sigma_{F}$ and $\frac{1}{\tau} \sum_{t \in T_{j}} \widetilde{F}_{t}^{(j)} \widetilde{F}_{t}^{(j) \prime}$. 
On step (2) we run an IV regression of $y_{i}=\frac{1}{T} \sum_{t=1}^{T} r_{i t}$ on the regressor

$$
x_{i}^{(1)}=\left(\begin{array}{c}
\widehat{\beta}_{i}^{(1)} \\
A_{1}\left(\widehat{\beta}_{i}^{(1)}-\widehat{\beta}_{i}^{(2)}\right)
\end{array}\right)=\left(\begin{array}{c}
\widehat{\beta}_{i}^{(1)} \\
A_{1} \frac{\eta_{1, T}-\eta_{2, T}}{\sqrt{\tau}} \mu_{i}+A_{1}\left(u_{i}^{(1)}-u_{i}^{(2)}\right)
\end{array}\right),
$$

with the instruments

$$
z_{i}^{(1)}=\left(\begin{array}{c}
\widehat{\beta}_{i}^{(3)} \\
\widehat{\beta}_{i}^{(3)}-\widehat{\beta}_{i}^{(4)}
\end{array}\right)=\left(\begin{array}{c}
\widehat{\beta}_{i}^{(3)} \\
\frac{\eta_{3, T}-\eta_{4, T}}{\sqrt{\tau}} \mu_{i}+\left(u_{i}^{(3)}-u_{i}^{(4)}\right)
\end{array}\right)
$$

The main estimation equation can be written in the following way:

$$
\begin{aligned}
y_{i} & =\frac{1}{T} \sum_{t \in T} r_{i t}=\widetilde{\lambda}^{\prime} \beta_{i}+\frac{\eta_{v, T}^{\prime}}{\sqrt{T}} \mu_{i}+\bar{e}_{i}=\widetilde{\lambda}^{\prime} \widehat{\beta}_{i}^{(1)}+\left(\frac{\eta_{v, T}^{\prime}}{\sqrt{T}}-\widetilde{\lambda}^{\prime} \frac{\eta_{1, T}}{\sqrt{\tau}}\right) \mu_{i}+\bar{e}_{i}-\widetilde{\lambda}^{\prime} u_{i}^{(1)} \\
& =\widetilde{\lambda}^{\prime} \widehat{\beta}_{i}^{(1)}+a_{1, T} A_{1}\left(\widehat{\beta}_{i}^{(1)}-\widehat{\beta}_{i}^{(2)}\right)+\bar{e}_{i}-\widetilde{\lambda}^{\prime} u_{i}^{(1)}-a_{1, T} A_{1}\left(u_{i}^{(1)}-u_{i}^{(2)}\right) .
\end{aligned}
$$

Thus, we can write it as follows:

$$
y_{i}=\left(\widetilde{\lambda}^{\prime}, a_{1, T}\right) x_{i}^{(1)}+\epsilon_{i}^{(1)} .
$$

Here we use the following notation:

$$
a_{1, T}=\left(\frac{\eta_{v, T}^{\prime}}{\sqrt{T}}-\widetilde{\lambda}^{\prime} \frac{\eta_{1, T}}{\sqrt{\tau}}\right)\left(A_{1} \frac{\eta_{1, T}-\eta_{2, T}}{\sqrt{\tau}}\right)^{-1} \Rightarrow\left(\frac{\eta_{v}^{\prime}}{2}-\eta_{1}\right)\left(A_{1}\left(\eta_{1}-\eta_{2}\right)\right)^{-1},
$$

and $\epsilon_{i}^{(1)}=\bar{e}_{i}-\tilde{\lambda}^{\prime} u_{i}^{(1)}-a_{1, T} A_{1}\left(u_{i}^{(1)}-u_{i}^{(2)}\right)$. Notice that $a_{1, T}$ is a random $1 \times k_{v}$ matrix that is well defined with probability approaching 1 (as $\eta_{1, T}$ and $\eta_{2, T}$ weakly converge to two independent random gaussian matrices), and $a_{1, T}$ is asymptotically of order $O_{p}(1)$.

The estimator computed on the step (2) of the four-split algorithm is

$$
\widehat{\lambda}^{(1)}=\left(I_{k_{F}}, 0_{k_{F}, k_{v}}\right)\left(X^{(1) \prime} Z^{(1)}\left(Z^{(1) \prime} Z^{(1)}\right)^{-1} Z^{(1) \prime} X^{(1)}\right)^{-1} X^{(1) \prime} Z^{(1)}\left(Z^{(1) \prime} Z^{(1)}\right)^{-1} Z^{(1) \prime} Y .
$$

Using equation (13) we obtain:

$$
\widehat{\lambda}^{(1)}-\tilde{\lambda}=\left(I_{k_{F}}, 0_{k_{F}, k_{v}}\right)\left(X^{(1) \prime} P_{Z^{(1)}} X^{(1)}\right)^{-1} X^{(1) \prime} P_{Z^{(1)}} \epsilon^{(1)},
$$

where $P_{Z}$ is a projection matrix onto $Z$. Let us introduce two normalizing matrices:

$$
Q_{x}=\left(\begin{array}{cc}
Q_{T} & 0_{k_{F}, k_{v}} \\
0_{k_{v}, k_{F}} & \sqrt{T} I_{k_{v}}
\end{array}\right), \quad Q_{z}=\left(\begin{array}{cc}
Q_{T} & 0_{k_{F}, k_{F}} \\
0_{k_{F}, k_{F}} & \sqrt{T} I_{k_{F}}
\end{array}\right) .
$$


The dimensionality of $Q_{x}$ is $k \times k$, where $k=k_{F}+k_{v}$ is a number of regressors in the second stage regression, while the dimensionality of $Q_{z}$ is $2 k_{F} \times 2 k_{F}$, where $2 k_{F}$ is the number of instruments. The matrix $Q_{T}$ was defined in the proof of Theorem 1. Now,

$$
Q_{x} x_{i}^{(1)}=\left(\widetilde{A}_{1, T} \gamma_{i}+\left(\begin{array}{cc}
Q_{T} & 0_{k_{F}, k_{F}} \\
\sqrt{T} A_{1} & -\sqrt{T} A_{1}
\end{array}\right)\left(\begin{array}{c}
u_{i}^{(1)} \\
u_{i}^{(2)}
\end{array}\right)\right),
$$

where

$$
\begin{aligned}
\widetilde{A}_{1, T}=\left(\begin{array}{cc}
I_{k_{F}} & Q_{T} \frac{\eta_{1, T}}{\sqrt{\tau}} \\
0_{k_{v}, k_{F}} & 2 A_{1}\left(\eta_{1, T}-\eta_{2, T}\right)
\end{array}\right) & \Rightarrow\left(\begin{array}{cc}
I_{k_{F}} & 2 \mathcal{I}_{k_{2}} \eta_{1} \\
0_{k_{v}, k_{F}} & 2 A_{1}\left(\eta_{1}-\eta_{2}\right)
\end{array}\right)=\widetilde{A}_{1}, \\
\frac{1}{\sqrt{T}}\left(\begin{array}{cc}
Q_{T} & 0_{k_{F}, k_{F}} \\
\sqrt{T} A_{1} & -\sqrt{T} A_{1}
\end{array}\right) & \rightarrow\left(\begin{array}{cc}
\mathcal{I}_{k_{2}} & 0_{k_{F}, k_{F}} \\
A_{1} & -A_{1}
\end{array}\right) .
\end{aligned}
$$

Here $\mathcal{I}_{k_{2}}$ is a $k_{F} \times k_{F}$ matrix which was introduced in Theorem 11. We also have

$$
Q_{z} z_{i}^{(1)}=\left(A_{1, T}^{*} \gamma_{i}+\left(\begin{array}{cc}
Q_{T} / \sqrt{T} & 0_{k_{F}, k_{F}} \\
I_{k_{F}} & -I_{k_{F}}
\end{array}\right) \sqrt{T}\left(\begin{array}{c}
u_{i}^{(3)} \\
u_{i}^{(4)}
\end{array}\right)\right),
$$

where

$$
\begin{aligned}
A_{1, T}^{*}=\left(\begin{array}{cc}
I_{k_{F}} & Q_{T} \frac{\eta_{3, T}}{\sqrt{\tau}} \\
0_{k_{F}, k_{F}} & 2\left(\eta_{3, T}-\eta_{4, T}\right)
\end{array}\right) & \Rightarrow\left(\begin{array}{cc}
I_{k_{F}} & 2 \mathcal{I}_{k_{2}} \eta_{3} \\
0_{k_{F}, k_{F}} & 2\left(\eta_{3}-\eta_{4}\right)
\end{array}\right)=A_{1}^{*}, \\
\left(\begin{array}{cc}
Q_{T} / \sqrt{T} & 0_{k_{F}, k_{F}} \\
I_{k_{F}} & -I_{k_{F}}
\end{array}\right) & \rightarrow\left(\begin{array}{cc}
\mathcal{I}_{k_{2}} & 0_{k_{F}, k_{F}} \\
I_{k_{F}} & -I_{k_{F}}
\end{array}\right) .
\end{aligned}
$$

Statements (1) and (5) of Lemma S1 imply that

$$
\begin{aligned}
\frac{T}{\sqrt{N}} \sum_{i=1}^{N} u_{i}^{(j)} u_{i}^{\left(j^{*}\right) \prime} & =O_{p}(1) \quad \text { for } \quad j \neq j^{*}, \\
\sqrt{\frac{T}{N}} \sum_{i=1}^{N}\left(\gamma_{i}^{\prime}, 1\right)^{\prime} u_{i}^{\left(j^{*}\right) \prime} & =O_{p}(1) .
\end{aligned}
$$

This together with Assumption LOADINGS gives us that

$$
\frac{1}{N} \sum_{i=1}^{N} Q_{x} x_{i}^{(1)} z_{i}^{(1) \prime} Q_{z} \Rightarrow \widetilde{A}_{1} \Gamma A_{1}^{* \prime} .
$$

By Assumption LOADINGS, $\Gamma$ is full rank, while $\widetilde{A}_{1}$ and $A_{1}^{* \prime}$ are full rank with probability 
1. Statements (3) and (4) of Lemma S1 imply that

$$
\frac{\tau}{N} \sum_{i=1}^{N} u_{i}^{(j)} u_{i}^{(j) \prime} \rightarrow^{p} \Sigma_{u}
$$

Thus, we obtain

$$
\begin{aligned}
& \frac{1}{N} \sum_{i=1}^{N} Q_{z} z_{i}^{(1)} z_{i}^{(1) \prime} Q_{z} \Rightarrow A_{1}^{*} \Gamma A_{1}^{* \prime}+4\left(\begin{array}{cc}
\mathcal{I}_{k_{2}} & 0_{k_{F}, k_{F}} \\
I_{k_{F}} & -I_{k_{F}}
\end{array}\right)\left(\begin{array}{cc}
\Sigma_{u} & 0_{k_{F}, k_{F}} \\
0_{k_{F}, k_{F}} & \Sigma_{u}
\end{array}\right)\left(\begin{array}{cc}
\mathcal{I}_{k_{2}} & I_{k_{F}} \\
0_{k_{F}, k_{F}} & -I_{k_{F}}
\end{array}\right) \\
& =A_{1}^{*} \Gamma A_{1}^{* \prime}+4\left(\begin{array}{cc}
\mathcal{I}_{k_{2}} \Sigma_{u} \mathcal{I}_{k_{2}} & \mathcal{I}_{k_{2}} \Sigma_{u} \\
\Sigma_{u} \mathcal{I}_{k_{2}} & 2 \Sigma_{u}
\end{array}\right) .
\end{aligned}
$$

Let us now show that

$$
\sqrt{\frac{T}{N}} \sum_{i=1}^{N} Q_{z} z_{i}^{(1)} \epsilon_{i}^{(1)}=O_{p}(1)
$$

We have $\epsilon_{i}^{(1)}=\bar{e}_{i}-\widetilde{\lambda}^{\prime} u_{i}^{(1)}-a_{1, T} A_{1}\left(u_{i}^{(1)}-u_{i}^{(2)}\right)$. The sum in (20) contains summands of the form $\sqrt{\frac{T}{N}} \sum_{i=1}^{N} \gamma_{i}\left(\bar{e}_{i}, u_{i}^{(j)}\right), \frac{T}{\sqrt{N}} \sum_{i=1}^{N} \bar{e}_{i} u_{i}^{(j)}$ and $\frac{T}{\sqrt{N}} \sum_{i=1}^{N} u_{i}^{\left(j^{*}\right) \prime} u_{i}^{(j)}$. All three types of summands are $O_{p}(1)$ due to statements (5), (2) and (1) of Lemma S1, correspondingly. Putting equations (17) and (19) together, we obtain

$$
\begin{gathered}
N Q_{x}^{-1} \Theta_{N, T, 1} Q_{z}^{-1}=\left(\frac{Q_{x} X^{(1) \prime} Z^{(1)} Q_{z}}{N}\left(\frac{Q_{z} Z^{(1) \prime} Z^{(1)} Q_{z}}{N}\right)^{-1} \frac{Q_{z} Z^{(1) \prime} X^{(1)} Q_{x}}{N}\right)^{-1} \\
. \frac{Q_{x} X^{(1) \prime} Z^{(1)} Q_{z}}{N}\left(\frac{Q_{z} Z^{(1) \prime} Z^{(1)} Q_{z}}{N}\right)^{-1}=O_{p}(1) .
\end{gathered}
$$

Putting everything together, we have:

$$
\sqrt{N T} Q_{T}^{-1}\left(\widehat{\lambda}^{(1)}-\widetilde{\lambda}\right)=\left(I_{k_{F}}, 0_{k_{F}, k_{v}}\right) N Q_{x}^{-1} \Theta_{N, T, 1} Q_{z}^{-1} \sqrt{\frac{T}{N}} \sum_{i=1}^{N} Q_{z} z_{i}^{(1)} \epsilon_{i}^{(1)}=O_{p}(1) .
$$

Because $\sqrt{N T} Q_{T}^{-1}=\left(\begin{array}{cc}\sqrt{N T} I_{k_{1}} & 0_{k_{1}, k_{2}} \\ 0_{k_{2}, k_{1}} & \sqrt{N} I_{k_{2}}\end{array}\right)$, we obtain different rates of estimation of the risk premia $\lambda_{1}$ and $\lambda_{2}$ on the strong and weak observed factors. We have $\sqrt{N T}\left(\widehat{\lambda}_{1}^{(1)}-\right.$ $\left.\widetilde{\lambda}_{1}\right)=O_{p}(1)$, while $\sqrt{N}\left(\widehat{\lambda}_{2}^{(1)}-\widetilde{\lambda}_{2}\right)=O_{p}(1)$. Thus,

$\sqrt{T}\left(\widehat{\lambda}_{1}^{(1)}-\lambda_{1}\right)=\sqrt{T}\left(\widetilde{\lambda}_{1}-\lambda_{1}\right)+\sqrt{T}\left(\widehat{\lambda}_{1}^{(1)}-\widetilde{\lambda}_{1}\right)=\sqrt{T}\left(\widetilde{\lambda}_{1}-\lambda_{1}\right)+O_{p}(1 / \sqrt{N}) \Rightarrow N\left(0, \Omega_{F}\right)$. 
As for the estimator of the risk premia on the weak factors,

$$
\widehat{\lambda}_{2}^{(1)}-\lambda_{2}=\left(\widetilde{\lambda}_{2}-\lambda_{2}\right)+\left(\widehat{\lambda}_{2}^{(1)}-\widetilde{\lambda}_{2}\right)=O_{p}(1 / \sqrt{T})+O_{p}(1 / \sqrt{N})=O_{p}(1 / \sqrt{\min \{N, T\}}) .
$$

We have proved the statement of Theorem 2 for an estimator obtained on step (2) of the algorithm, but the same line of reasoning applies to $\widehat{\lambda}^{(2)}, \widehat{\lambda}^{(3)}, \widehat{\lambda}^{(4)}$ and their average. This finishes the proof of Theorem 2 .

Proof of Theorem 3. Following the steps of the proof of Theorem 2 we get the following two statements:

$$
\begin{aligned}
& \frac{1}{N} \sum_{i=1}^{N} Q_{x} x_{i}^{(j)} z_{i}^{(j) \prime} Q_{z} \Rightarrow \widetilde{A}_{j} \boldsymbol{\Gamma} A_{j}^{* \prime}, \\
& \frac{1}{N} \sum_{i=1}^{N} Q_{z} z_{i}^{(j)} z_{i}^{(j) \prime} Q_{z} \Rightarrow A_{j}^{*} \Gamma A_{j}^{* \prime}+4\left(\begin{array}{cc}
\mathcal{I}_{k_{2}} \Sigma_{u} \mathcal{I}_{k_{2}} & \mathcal{I}_{k_{2}} \Sigma_{u} \\
\Sigma_{u} \mathcal{I}_{k_{2}} & 2 \Sigma_{u}
\end{array}\right),
\end{aligned}
$$

where $A_{j}^{*}$ and $\widetilde{A}_{j}$ are random matrices that are deterministic functions of random vectors $\left(\eta_{1}, \ldots, \eta_{4}\right)$. Indeed, let us adopt the following notation. Let $j_{1}, \ldots, j_{4}$ be the circular indexes used for computing $\widehat{\lambda}^{(j)}$. In particular, the estimate $\widehat{\lambda}^{(j)}$ is computed from the IV regression with the regressors $x_{i}^{(j)}=\left(\widehat{\beta}_{i}^{\left(j_{1}\right) \prime},\left(\widehat{\beta}_{i}^{\left(j_{1}\right)}-\widehat{\beta}_{i}^{\left(j_{2}\right)}\right)^{\prime} A_{j}^{\prime}\right)^{\prime}$ and the instruments $z_{i}^{(j)}=\left(\widehat{\beta}_{i}^{\left(j_{3}\right) \prime},\left(\widehat{\beta}_{i}^{\left(j_{3}\right)}-\widehat{\beta}_{i}^{\left(j_{4}\right)}\right)^{\prime}\right)^{\prime}$. Then, similarly to the proof of Theorem 2 , we obtain:

$$
A_{j}^{*}=\left(\begin{array}{cc}
I_{k_{F}} & 2 \mathcal{I}_{k_{2}} \eta_{j_{3}} \\
0_{k_{F}, k_{F}} & 2\left(\eta_{j_{3}}-\eta_{j_{4}}\right)
\end{array}\right), \quad \widetilde{A}_{j}=\left(\begin{array}{cc}
I_{k_{F}} & 2 \mathcal{I}_{k_{2}} \eta_{j_{1}} \\
0_{k_{v}, k_{F}} & 2 A_{j}\left(\eta_{j_{1}}-\eta_{j_{2}}\right)
\end{array}\right) .
$$

So,

$$
N Q_{x}^{-1}\left(X^{(j) \prime} Z^{(j)}\left(Z^{(j) \prime} Z^{(j)}\right)^{-1} Z^{(j) \prime} X^{(j)}\right)^{-1} X^{(j) \prime} Z^{(j)}\left(Z^{(j) \prime} Z^{(j)}\right)^{-1} Q_{z}^{-1} \Rightarrow \Theta_{j}
$$

The limit $\Theta_{j}$ in the last expression is a known deterministic function of random vectors $\left(\eta_{1}, \ldots, \eta_{4}\right)$, which can be explicitly written in terms of $A_{j}^{*}$ and $\widetilde{A}_{j}$.

We have the following expression for the estimates obtained on steps (2) and (3) of the four-split algorithm:

$$
\sqrt{N T} Q_{T}^{-1}\left(\widehat{\lambda}^{(j)}-\tilde{\lambda}\right)=\left(I_{k_{F}}, 0_{k_{F}, k_{v}}\right) N Q_{x}^{-1} \Theta_{N, T, j} Q_{z}^{-1} \sqrt{\frac{T}{N}} \sum_{i=1}^{N} Q_{z} z_{i}^{(j)} \epsilon_{i}^{(j)},
$$


where $\epsilon_{i}^{(j)}=\bar{e}_{i}-\widetilde{\lambda}^{\prime} u_{i}^{\left(j_{1}\right)}-a_{j, T} A_{j}\left(u_{i}^{\left(j_{1}\right)}-u_{i}^{\left(j_{2}\right)}\right)$, and

$$
Q_{z} z_{i}^{(j)}=A_{j, T}^{*} \gamma_{i}+\left(\begin{array}{cc}
Q_{T} / \sqrt{T} & 0_{k_{F}, k_{F}} \\
I_{k_{F}} & -I_{k_{F}}
\end{array}\right) \sqrt{T}\left(\begin{array}{c}
u_{i}^{\left(j_{3}\right)} \\
u_{i}^{\left(j_{4}\right)}
\end{array}\right)
$$

Consider the following term which can be rewritten in terms of $\xi_{i}$ from Assumption GAUSSIANITY:

$$
\begin{aligned}
& \sqrt{\frac{T}{N}} \sum_{i=1}^{N} Q_{z} z_{i}^{(j)} \epsilon_{i}^{(j)}=A_{j, T}^{*}\left(\sqrt{\frac{T}{N}} \sum_{i=1}^{N} \gamma_{i}\left(\begin{array}{c}
\bar{e}_{i} \\
u_{i}^{\left(j_{1}\right)} \\
u_{i}^{\left(j_{2}\right)}
\end{array}\right)^{\prime}\right)\left(\begin{array}{c}
1 \\
-\widetilde{\lambda}-A_{j}^{\prime} a_{j, T}^{\prime} \\
A_{j}^{\prime} a_{j, T}^{\prime}
\end{array}\right) \\
& +\left(\begin{array}{cc}
Q_{T} / \sqrt{T} & 0_{k_{F}, k_{F}} \\
I_{k_{F}} & -I_{k_{F}}
\end{array}\right)\left(\frac{T}{\sqrt{N}} \sum_{i=1}^{N}\left(\begin{array}{c}
u_{i}^{\left(j_{3}\right)} \\
u_{i}^{\left(j_{4}\right)}
\end{array}\right)\left(\begin{array}{c}
\bar{e}_{i} \\
u_{i}^{\left(j_{1}\right)} \\
u_{i}^{\left(j_{2}\right)}
\end{array}\right)^{\prime}\right)\left(\begin{array}{c}
1 \\
-\widetilde{\lambda}-A_{j}^{\prime} a_{j, T}^{\prime} \\
A_{j}^{\prime} a_{j, T}^{\prime}
\end{array}\right) \\
& =\mathcal{A}_{j, T} \frac{1}{\sqrt{N}} \sum_{i=1}^{N} \xi_{i},
\end{aligned}
$$

where $\mathcal{A}_{j, T}$ is a $k_{z} \times k_{\xi}$ matrix which is a deterministic function of $A_{j, T}^{*}, A_{j}, a_{j, T}, \tilde{\lambda}$. The exact expression for $\mathcal{A}_{j, T}$ is obvious though too complicated to write down. We have discussed before the convergence of all terms separately, which implies that $\mathcal{A}_{j, T} \Rightarrow \mathcal{A}_{j}$, where the limit is a deterministic function of $\left(\eta_{1}, \ldots, \eta_{4}\right)$.

Given Assumption GAUSSIANITY, we have $\sqrt{\frac{T}{N}} \sum_{i=1}^{N} Q_{z} z_{i}^{(j)} \epsilon_{i}^{(j)} \Rightarrow \mathcal{A}_{j} \xi$. Following step (4) of the four-split algorithm, we can put all pieces together:

$$
\sqrt{N T} Q^{-1}\left(\widehat{\lambda}_{4 S}-\widetilde{\lambda}\right) \Rightarrow\left(I_{k_{F}}, 0_{k_{F}, k_{v}}\right)\left(\frac{1}{4} \sum_{j=1}^{4} \Theta_{j} \mathcal{A}_{j}\right) \xi
$$

As we can see, the four-split estimator is asymptotically mixed gaussian; that is, the limit distribution conditionally on $\eta_{1}, \ldots, \eta_{4}$ (which is independent of $\xi$ due to Assumption ERRORS) is gaussian with mean zero and the variance depending on $\eta_{1}, \ldots, \eta_{4}$.

Denote $\widehat{\Sigma}_{I V}=\frac{1}{N} R^{\prime} G^{-1} \widehat{\Sigma}_{0} G^{-1} R$. We show below that $\widehat{\Sigma}_{I V}$ has the following asymptotic distribution:

$$
N T Q_{T}^{-1} \widehat{\Sigma}_{I V} Q_{T}^{-1} \Rightarrow\left(I_{k_{F}}, 0_{k_{F}, k_{v}}\right)\left(\frac{1}{4} \sum_{j=1}^{4} \Theta_{j} \mathcal{A}_{j}\right) \Sigma_{\xi}\left(\frac{1}{4} \sum_{j=1}^{4} \Theta_{j} \mathcal{A}_{j}\right)^{\prime}\left(I_{k_{F}}, 0_{k_{F}, k_{v}}\right)^{\prime} .
$$

Statement (24) implies the statement of Theorem 3. Indeed, equations (23) and (24) 
imply that $\widehat{\Sigma}_{I V}^{-1 / 2}\left(\widehat{\lambda}_{4 S}-\widetilde{\lambda}\right) \Rightarrow N\left(0, I_{k}\right)$, where the limiting gaussian vector is independent of the limiting gaussian vector in the following convergence:

$$
\sqrt{T} \Omega_{F}^{-1 / 2}(\widetilde{\lambda}-\lambda) \Rightarrow N\left(0, I_{k}\right)
$$

The expression $\widehat{\Sigma}_{4 S}^{-1 / 2}\left(\widehat{\lambda}_{4 S}-\lambda\right)$ is the weighted sum of the expressions staying on the lefthand-side of the last two convergence with weights asymptotically independent from both limiting $N\left(0, I_{k}\right)$. This leads to the validity of the statement of Theorem 3 .

To prove the validity of statement (24) we notice that $\sqrt{T} Q_{z} z_{i}^{(j)} \epsilon_{i}^{(j)}=\mathcal{A}_{j, T} \xi_{i}$. Thus,

$$
\begin{aligned}
& \frac{T}{N} \sum_{i=1}^{N}\left(\begin{array}{c}
Q_{z} \widetilde{z}_{i}^{(1)} \epsilon_{i}^{(1)} \\
\ldots \\
Q_{z} \widetilde{z}_{i}^{(4)} \epsilon_{i}^{(4)}
\end{array}\right)\left(\begin{array}{c}
Q_{z} \widetilde{z}_{i}^{(1)} \epsilon_{i}^{(1)} \\
\ldots \\
Q_{z} \widetilde{z}_{i}^{(4)} \epsilon_{i}^{(4)}
\end{array}\right)^{\prime}=\left(\begin{array}{c}
\mathcal{A}_{1, T} \\
\ldots \\
\mathcal{A}_{4, T}
\end{array}\right) \frac{1}{N} \sum_{i=1}^{N} \xi_{i} \xi_{i}^{\prime}\left(\begin{array}{c}
\mathcal{A}_{1, T} \\
\ldots \\
\mathcal{A}_{4, T}
\end{array}\right)^{\prime} \\
& \Rightarrow\left(\begin{array}{c}
\mathcal{A}_{1} \\
\ldots \\
\mathcal{A}_{4}
\end{array}\right) \Sigma_{\xi}\left(\begin{array}{c}
\mathcal{A}_{1} \\
\ldots \\
\mathcal{A}_{4}
\end{array}\right)^{\prime}
\end{aligned}
$$

Let us consider an infeasible variance estimator $\widetilde{\Sigma}_{I V}$ which is constructed in the same way as $\widehat{\Sigma}_{I V}$ but uses $\epsilon_{i}^{(j)}$ in place of $\widehat{\epsilon}_{i}^{(j)}$. That is, denote

$$
\widetilde{\Sigma}_{0}=\frac{1}{N} \sum_{i=1}^{N}\left(\begin{array}{c}
\widetilde{z}_{i}^{(1)} \epsilon_{i}^{(1)} \\
\ldots \\
\widetilde{z}_{i}^{(4)} \epsilon_{i}^{(4)}
\end{array}\right)\left(\begin{array}{c}
\widetilde{z}_{i}^{(1)} \epsilon_{i}^{(1)} \\
\ldots \\
\widetilde{z}_{i}^{(4)} \epsilon_{i}^{(4)}
\end{array}\right)^{\prime}
$$

and consider $\widetilde{\Sigma}_{I V}=\frac{1}{N} R^{\prime} G^{-1} \widetilde{\Sigma}_{0} G^{-1} R$. By putting together (21), (22) and (25) we obtain

$$
N T Q_{T}^{-1} \widetilde{\Sigma}_{I V} Q_{T}^{-1} \Rightarrow\left(I_{k_{F}}, 0_{k_{F}, k_{v}}\right)\left(\frac{1}{4} \sum_{j=1}^{4} \Theta_{j} \mathcal{A}_{j}\right) \Sigma_{\xi}\left(\frac{1}{4} \sum_{j=1}^{4} \Theta_{j} \mathcal{A}_{j}\right)^{\prime}\left(I_{k_{F}}, 0_{k_{F}, k_{v}}\right)^{\prime} .
$$

The only thing left to show is that the difference between $\widehat{\Sigma}_{I V}$ and $\widetilde{\Sigma}_{I V}$ is asymptotically negligible. In particular, we will show for any $j$ and $j^{*}$,

$$
\frac{T}{N} \sum_{i=1}^{N} Q_{z} z_{i}^{(j)} z_{i}^{\left(j^{*}\right) \prime} Q_{z}\left(\epsilon_{i}^{(j)} \epsilon_{i}^{\left(j^{*}\right)}-\widehat{\epsilon}_{i}^{(j)} \widehat{\epsilon}_{i}^{\left(j^{*}\right)}\right) \rightarrow^{p} 0
$$

where $\widehat{\epsilon}_{i}^{(j)}$ are the residuals from the $(j)^{\text {th }}$ IV regression. Indeed, this last statement implies that $\widehat{\Sigma}_{I V}=\widetilde{\Sigma}_{I V}\left(1+o_{p}(1)\right)$, and usage of residuals in place of true errors does not have 
an asymptotic effect on estimation of variance.

In order to prove (26) we write down an equation analogous to equation (13):

$$
y_{i}=\left(\widetilde{\lambda}^{\prime}, a_{j, T}\right) x_{i}^{(j)}+\epsilon_{i}^{(j)}=\theta_{j}^{\prime} x_{i}^{(j)}+\epsilon_{i}^{(j)} .
$$

From the proof of Theorem 2 we have that $\sqrt{N T} Q_{x}^{-1}\left(\widehat{\theta}_{j}-\theta_{j}\right)=O_{p}(1)$, where $\widehat{\theta}_{j}$ is the IV estimator obtained on Steps (2) for $j=1$ or on Step (3) for $j=2,3$ or 4 . The residuals for this regression are

$$
\widehat{\epsilon}_{i}^{(j)}=y_{i}-\widehat{\theta}_{j}^{\prime} x_{i}^{(j)}=\epsilon_{i}^{(j)}-\left(\widehat{\theta}_{j}-\theta_{j}\right)^{\prime} x_{i}^{(j)}=\epsilon_{i}^{(j)}-\left(Q_{x}^{-1}\left(\widehat{\theta}_{j}-\theta_{j}\right)\right)^{\prime} Q_{x} x_{i}^{(j)} .
$$

The left hand expression of (26) is equal to

$\frac{T}{N} \sum_{i=1}^{N} Q_{z} z_{i}^{(j)} z_{i}^{\left(j^{*}\right) \prime} Q_{z}\left(\epsilon_{i}^{(j)}\left(\widehat{\theta}_{j^{*}}-\theta_{j^{*}}\right)^{\prime} x_{i}^{\left(j^{*}\right)}+\epsilon_{i}^{\left(j^{*}\right)}\left(\widehat{\theta}_{j}-\theta_{j}\right)^{\prime} x_{i}^{(j)}-\left(\widehat{\theta}_{j^{*}}-\theta_{j^{*}}\right)^{\prime} x_{i}^{\left(j^{*}\right)}\left(\widehat{\theta}_{j}-\theta_{j}\right)^{\prime} x_{i}^{(j)}\right)$.

This expression contains three sums. We can show that each of them is asymptotically negligible. For example, consider the first of the three sums:

$$
\begin{array}{r}
\frac{1}{N^{3 / 2}} \sum_{i=1}^{N}\left(\sqrt{T} Q_{z} z_{i}^{(j)} \epsilon_{i}^{(j)}\right)\left(Q_{z} z_{i}^{\left(j^{*}\right)}\right)^{\prime}\left(Q_{x} x_{i}^{\left(j^{*}\right)}\right)^{\prime}\left(\sqrt{N T} Q_{x}^{-1}\left(\widehat{\theta}_{j^{*}}-\theta_{j^{*}}\right)\right) \\
\quad=\frac{1}{N^{3 / 2}} \sum_{i=1}^{N} \mathcal{A}_{j, T} \xi_{i}\left(Q_{z} z_{i}^{\left(j^{*}\right)}\right)^{\prime}\left(Q_{x} x_{i}^{\left(j^{*}\right)}\right)^{\prime}\left(\sqrt{N T} Q_{x}^{-1}\left(\widehat{\theta}_{j^{*}}-\theta_{j^{*}}\right)\right)
\end{array}
$$

Note that $\sqrt{N T} Q_{x}^{-1}\left(\widehat{\theta}_{j^{*}}-\theta_{j^{*}}\right)=O_{p}(1)$. As before, $Q_{z} z_{i}^{(j)}=O_{p}(1) \gamma_{i}+O_{p}(1) \sqrt{T}\left(u_{i}^{\left(j_{3}\right)}, u_{i}^{\left(j_{4}\right)}\right)^{\prime}$, while $Q_{x} x_{i}^{(j)}=O_{p}(1) \gamma_{i}+O_{p}(1) \sqrt{T}\left(u_{i}^{\left(j_{1}\right)}, u_{i}^{\left(j_{2}\right)}\right)$, where all the mentioned $O_{p}(1)$ terms are not indexed by $i$. Thus,

$$
\frac{1}{N^{3 / 2}} \sum_{i=1}^{N} \mathcal{A}_{j, T} \xi_{i}\left(Q_{z} z_{i}^{\left(j^{*}\right)}\right)^{\prime}\left(Q_{x} x_{i}^{\left(j^{*}\right)}\right)^{\prime}=O_{p}(1) \frac{1}{N^{3 / 2}} \sum_{i=1}^{N} \xi_{i} \xi_{i}^{\prime}+O_{p}(1) \frac{1}{N^{3 / 2}} \sum_{i=1}^{N} \xi_{i} \otimes\left(\gamma_{i} \gamma_{i}^{\prime}\right)
$$

By Assumption GAUSSIANITY, $\frac{1}{N^{3 / 2}} \sum_{i=1}^{N} \xi_{i} \xi_{i}^{\prime} \rightarrow^{p} 0$ and thus

$$
\frac{1}{N^{3 / 2}}\left\|\sum_{i=1}^{N} \xi_{i} \otimes\left(\gamma_{i} \gamma_{i}^{\prime}\right)\right\| \leq \frac{1}{N^{3 / 2}} \sqrt{\sum_{i=1}^{N}\left\|\xi_{i}\right\|^{2}} \sqrt{\sum_{i=1}^{N}\left\|\gamma_{i}\right\|^{4}} \rightarrow^{p} 0 .
$$

This gives the asymptotic negligibility of the first sum; the negligibility of the other two sums is proved in a similar manner. This ends the proof of Theorem 3 . 